\title{
Effective stress concept in unsaturated soils: Clarification and validation of a unified framework
}

\author{
Mathieu Nuth ${ }^{* \dagger}$ and Lyesse Laloui \\ Soil Mechanics Laboratory, Ecole Polytechnique Fédérale de Lausanne, EPFL, Switzerland
}

\begin{abstract}
SUMMARY
The effective stress principle, conventionally applied in saturated soils, is reviewed for constitutive modelling purposes. The assumptions for the applicability of Terzaghi's single effective stress are recalled and its advantages are inventoried. The possible stress frameworks applicable to unsaturated soil modelling are reassessed in a comparative manner, specifically the Bishop's single effective stress, the independent stress variables approach and the generalized stress framework. The latter considerations lead to the definition of a unified stress context, suitable for modelling soils under different saturation states. In order to qualify the implications brought by the proposed stress framework, several experimental data sets are re-examined in the light of the generalized effective stress. The critical state lines (CSLs) at different saturation states tend to converge remarkably towards a unique saturated line in the deviatoric stress versus mean effective stress plane. The effective stress interpretation is also applied to isotropic paths and compared with conventional net stress conception. The accent is finally laid on a second key feature for constitutive frameworks based on a unified stress, namely the sufficiency of a unique mechanical yield surface besides the unique CSL. Copyright (C) 2007 John Wiley \& Sons, Ltd.
\end{abstract}

Received 28 November 2006; Revised 21 May 2007; Accepted 21 May 2007

KEY WORDS: effective stress; unsaturated soils; critical state; stress framework; constitutive modelling

\section{INTRODUCTION}

At early stages of soil mechanics, Terzaghi [1] introduced the concept of effective stress for the particular case of saturated soils followed by other major contributions to the definition of effective stress [2,3]. Since these early works, the effective stress principle has been widely used in modelling geotechnical engineering applications, giving a simple link between elastic deformation and stress acting in the soil, the latter being proportional to the observed deformation in saturated

\footnotetext{
${ }^{*}$ Correspondence to: Mathieu Nuth, LMS-ICARE, Station 18, EPFL, CH-1015, Lausanne, Switzerland.

${ }^{\dagger}$ E-mail: mathieu.nuth@epfl.ch

Contract/grant sponsor: Swiss State Secretariat for Education and Research SER; contract/grant number: OFES C03.0021-COST C15
} 
porous media. The effective stress is a combination of both the externally applied stresses and the internal pressure of fluid phase(s) and enables the conversion of a multiphase porous medium into mechanically equivalent single-phase continuum. Indeed, the stress state is considered as uniquely represented by the effective stress.

The objective of this contribution is to draw back the historic developments of the effective stress in the framework of porous materials, remaining as long as possible in a macroscopic conception of saturated or fully dry soil mechanics. Then, the accent is laid on the extension of the effective stress principle to materials saturated with more than one fluid. A particular attention is paid to the various stress frameworks elected up to now for constitutive modelling of unsaturated soils, by covering the early single effective stress, the independent stress variables approach and the most recent combined effective stress conceptions. The stress framework considerations developed below are only intended to introduce constitutive modelling of unsaturated soils. Nevertheless, some fundamental modelling aspects such as general constitutive equations or yield limit definition will be dealt with in the following.

Once the appropriate stress framework is elected regarding the analysis led throughout the paper, the major implications of the 'Bishop generalized effective stress framework' are eventually investigated. The principle is to re-plot experimental data in conventional stress-strain planes, stress variables being usually net stress and suction, into new effective stress planes. Isotropic compression, drying-wetting paths and shear results are examined in order to evidence possible simplifications brought by the use of the unified effective stress framework.

\section{CONCEPTUAL PRINCIPLE OF EFFECTIVE STRESS}

The need for unified formulations of the stress variables raised from the complexity of modelling the behaviour of porous materials, which are likely to be filled fully or partially with fluids of different nature from the skeleton matrix, termed 'solid phase' in the following. Several theories tend to reach a homogenization of the phases in order to obtain an equivalent macroscopic continuum. Then, the elastic or elastic-plastic deformations are supposed to be directly linked to changes in the macroscopic stress quantity.

Although the lexicon of effective stress is due to Terzaghi [1], as recalled in next paragraph, a voluntarily more general view is presented in this section. The effective stress concept is basically applicable to any porous medium that may undergo effects of both external loads and internal pore pressures, also called interstitial pressures, due to possibility of fluids within the pore space. Imitating Terzaghi's definition of the principle of effective stress, one can formulate two statements:

- Seating solely in the solid skeleton of the medium, the effective stress enters the elastic and elasto-plastic constitutive equations of the soil matrix, linking a change in stress to strain-like quantity of the skeleton. The effective stress can thus be simply defined as that inducing the mechanical elastic strain of the solid skeleton, leading to the following simple constitutive equation:

$$
\dot{\varepsilon}_{i j}^{\mathrm{e}}=C_{i j k l}^{\mathrm{e}} \dot{\sigma}_{k l}^{\prime}
$$

where $\dot{\varepsilon}_{i j}^{\mathrm{e}}$ is the rate of elastic strain of the solid skeleton, $C_{i j k l}^{\mathrm{e}}$ is the drained elastic compliance matrix and $\dot{\sigma}_{k l}^{\prime}$ the increment of effective stress. A unique stress is necessary and sufficient to describe the mechanical behaviour. 


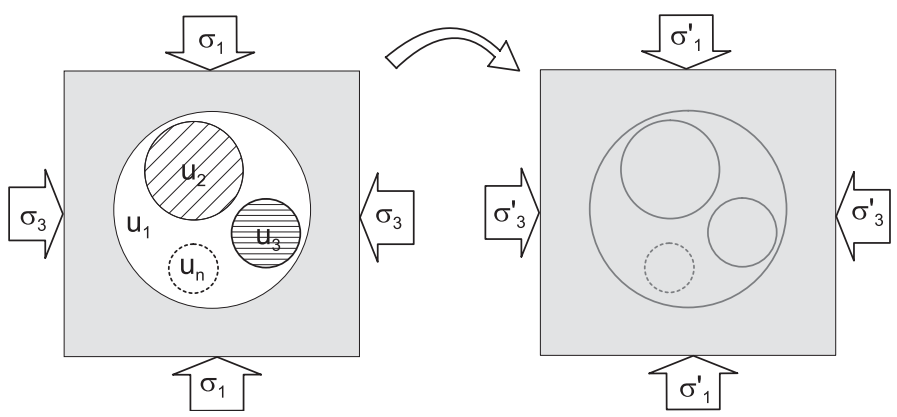

Figure 1. Conversion of multiphase and multistress medium (solid phase and pore space filled with $n$ fluids) into single continuum. $u_{\beta}$ designates the interstitial pressure of fluid $\beta, \sigma_{i j}$ is the exterior stress.

- Expressed as a function of the externally applied stresses and the internal pore fluid pressures, the effective stress converts the analysis of a multiphase porous medium into a mechanically equivalent, single-phase, single-stress state continuum comprehension.

In a similar manner to Khalili et al. [4], Figure 1 gives a schematic representation of the effective stress principle. The unified effective stress $\sigma_{i j}^{\prime}$ averaged over the total volume schemed in Figure 1 is written as follows:

$$
\sigma_{i j}^{\prime}=\sigma_{i j}-\sum_{\beta=1}^{n} \alpha_{\beta} u_{\beta} \delta_{i j}
$$

where $\sigma_{i j}$ designates the total exterior stress, $u_{\beta}$ the pressure of fluid $\beta$ and $\alpha_{\beta}$ the scaling factor for phase $\beta$. As developed in depth later, parameters $\alpha_{\beta}$ should account for the influence of each fluid pressure on the overall behaviour. The different fluid contributions are also likely to be linked to their respective volumetric fraction. Independently of the stress state, all the fluids, may they be wetting or not, are assumed to react in an isotropic way, generating the same pressure in all directions. Obviously, any supplementary interaction between the different fluid phases should be integrated in the generalized stress equation or at least accounted for in parallel to Equation (2).

The previous general definition is intended for the widest range of porous media or geomaterials, fluid phases being possibly liquid (e.g. water, oil) or gaseous (air). Furthermore, the generic equation (2) let the effective stress concept being applicable to the categories of materials classified between fully separated grains and solid rock with interconnected pores. However, no single effective stress formulation has ever been proved to be applicable to all materials at once; so, even though the effective stress concept remains applicable to many materials, the formulation of the stress variable itself (see coefficients $\alpha_{\beta}$ in Equation (2)) is very likely to vary from one structure to another. Nevertheless, a proper definition of the unified effective stress enables addressing several kinds of geomechanical problems. The range of applications could thus extend from the constitutive modelling of unsaturated soils to the interpretation of pollutants draining in geomaterials.

\section{OVERVIEW OF EFFECTIVE STRESS FORMULATIONS FOR SATURATED SOILS}

Historically, the general definition in the previous paragraph has been first written for the simple case of a porous medium filled with one single fluid [1]. Focusing on soils, two limit states of 

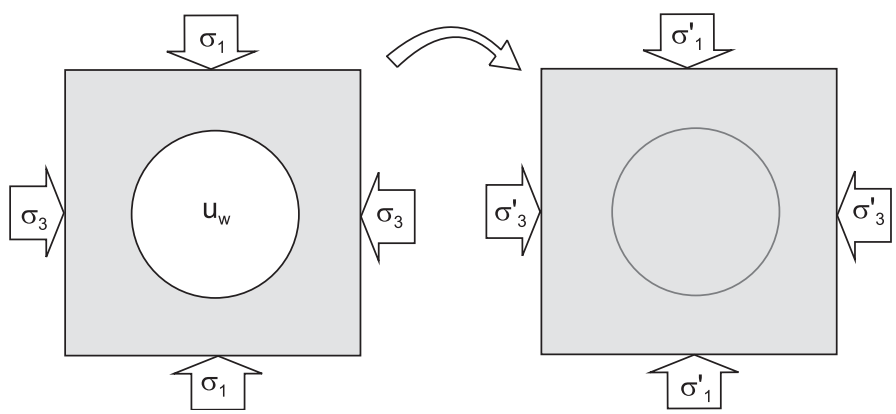

Figure 2. Graphical representation of effective stress concept for saturated soils.

saturation are remarkable. Firstly, interstitial space can be filled with air, assumed compressible. The effective stress in the dry soil is

$$
\sigma_{i j}^{\prime}=\sigma_{i j}
$$

Equation (3) is valid only if air pressure remains equal to the atmospheric (reference) pressure.

Secondly, Karl Terzaghi also writes a proper version of the effective stress principle for soils saturated with water (Figure 2), using the following two sentences [1]:

- All measurable effects of a change of stress of the soil, that is, compression, distortion and change of shearing resistance, are exclusively due to changes in effective stress.

- $\sigma_{i j}^{\prime}$ is defined by

$$
\sigma_{i j}^{\prime}=\sigma_{i j}-u_{\mathrm{w}} \delta_{i j}
$$

with $u_{\mathrm{w}}$ being the pore water pressure and $\delta_{i j}$ the Kroenecker's delta: $\delta_{i i}=1 ; \delta_{i \neq j}=0$. One assumption implicitly made here is the incompressibility of the fluid phase.

Terzaghi's expression for the effective stress was shown to be the 'experimental true form', in the sense that it allows an accurate comprehension of consolidation process among others. However, it is only representative of the very particular case of saturated soils with incompressible grains and a pore space completely filled with incompressible fluid.

Considering that the latter two assumptions could not be verified for the full range of geomaterials or with particular conditions, other formulations have been proposed for the effective stress for saturated materials. In the synthesized review of the main proposals presented hereafter, the effective stresses are written under the generic formulation of the following equation, which is obviously a particular form of Equation (2) limited to a single fluid phase:

$$
\sigma_{i j}^{\prime}=\sigma_{i j}-\alpha_{\mathrm{w}} u_{\mathrm{w}} \delta_{i j}
$$

In a voluntary effort to remain at a macroscopic level of stress, or at least to focus mostly on experimental true forms of skeleton stresses, any considerations implying microscopic or grain-size analyses (e.g. $[2,5])$ are disregarded here.

In fact, Terzaghi himself first suggested that $\alpha_{\mathrm{w}}$ should equal the material porosity $n$, but ended up with the final equation (4) based on the experimental observation. Nevertheless, Biot [6], among others, argued that the effect of the interstitial pressure $u_{\mathrm{w}}$ should actually be scaled down 
in Terzaghi's expression in order to weight the respective reactions proportionally to the volumetric fractions. This is in agreement with the preliminary comments stated about Equation (2). A possible form of the effective stress could include the porosity $n$, which is the volume of voids over the total volume, as a scaling factor for the fluid pressure, leading to the form

$$
\sigma_{i j}^{\prime}=\sigma_{i j}-n u_{\mathrm{w}} \delta_{i j}
$$

where one volumetric state parameter is required to be known.

From a different point of view, the grain compressibility is not always negligible versus that of the skeleton. Coefficient $\alpha_{\mathrm{w}}$ in Equation (5) is thus assumed by Skempton [2] to depend on the finite compressibility of the grains $C_{\mathrm{s}}$ and the drained compressibility of the granular skeleton $C$.

$$
\sigma_{i j}^{\prime}=\sigma_{i j}-\left(1-\frac{C_{\mathrm{s}}}{C}\right) u_{\mathrm{w}} \delta_{i j}
$$

Nur and Byerlee [3], among others, determined identically $\alpha_{\mathrm{w}}=\left(1-C_{\mathrm{s}} / C\right)$ in the particular framework of rock mechanics.

The last main family of effective stresses for saturated soils makes use of both material parameters cited previously, coefficient $\alpha_{\mathrm{w}}$ being a combination of porosity and compressibility coefficients. For instance, Suklje [7] proposed the following formulation:

$$
\alpha_{\mathrm{w}}=1-(1-n) \frac{C}{C_{\mathrm{s}}}
$$

As a conclusion, the general form of effective stress for saturated soils (5) is declined into several main families, involving either the porosity, the compressibility or both, as reviewed by Jardine et al. [8]. Letting extra parameters appear in the effective stress may enable a more general comprehension of natural materials, considering that separated grains may behave differently than solid rock. However, some basic assumptions often enable a neat simplification of the effective stress form. This does not mean that a unique effective stress applicable to all materials could ever be determined. Indeed, in the case of soils, the assumption of a negligible compressibility of grains versus that of the whole skeleton is mostly reasonable, as evaluated by Skempton [2], and enables recovering Terzaghi's effective stress (4). Hence, in the following, accepting the assumptions of incompressibility of water and solid grains mentioned previously, Terzaghi's effective stress is believed to represent the only stress governing the mechanical elastic strain of the soil material in saturated conditions. Assumptions on compressibility are supposed to hold for the unsaturated conditions.

\section{REVIEW OF STRESS FRAMEWORKS FOR UNSATURATED SOILS}

Even though the long-time debate on the most appropriate stress framework for unsaturated soils has eventually come to an end, evidencing the need for complete hydro-mechanical stress framework $[8,9]$, several forms of adequate stress variables are still possible. Those different unified stresses, now used for advanced constitutive modelling of unsaturated soils, present several levels of complexity and can be considered as inherited either from the Bishop single effective stress or from the independent stress variable approach. The common feature of the new stress conceptions is nevertheless the use of suction, or a modified version of it, as a second stress variable to build 
complete hydro-mechanical frameworks. It is proposed hereafter to overview the arguments at the origin of these advanced unified stresses, by retracing the chronological evolution of effective, independent and combined stresses for unsaturated soil modelling.

\subsection{Bishop single effective stress}

4.1.1. A single effective stress for unsaturated soils. Partial saturation, corresponding to more than one fluid filling the pores, is considered as the most common state for natural and engineered soils. As Terzaghi's effective stress is valid only for the limit states of full saturation of pores with one fluid alone, namely water or air, a need for extending the effective stress principle to unsaturated states raised. In the present case, the soil is a porous medium saturated with two fluids, one being a wetting fluid with respect to the other nonwetting fluid, respectively, water and air, for instance. In natural conditions, the water is obviously a mixture of water and dissolved air rather than pure water. Reversely, the gaseous phase can be almost saturated with water. In the following, unless otherwise specified, each of the two fluid phases is assumed to be homogeneous as idealized liquid and gaseous fluids, respectively. In comparison with the bi-phasic problem previously treated - solid grains and water-the third phase appearing in the pore space cannot be considered as uncompressible. This basically gives birth to a specific behaviour of the interstitial fluids as well as particular fluid-fluid interfaces.

In the early works of Bishop [10], the effective stress in unsaturated soils has been straightforwardly defined as 'a function of the total stress and the pore pressure which controls the mechanical effects of a change in stress', the goal being once more to convert a multiphase and multistress medium into a mechanically equivalent single phase and stress state continuum. In other words, extending Terzaghi's proposals to partially saturated soils means assuming that:

- All measurable effects of a change of stress of the soil are exclusively due to changes in the effective stress.

- In unsaturated soils, the effective stress is defined as the excess of total stress $\sigma_{i j}$ over an equivalent pore pressure $u^{*}$ :

$$
\sigma_{i j}^{\prime}=\sigma_{i j}-u^{*} \delta_{i j}
$$

The quantity $u^{*}$ may be considered as that portion of the effective stress in a soil resulting from the pressure of all fluids in the pores. Defining $u_{\mathrm{a}}$ as the interstitial air pressure in addition to the pore water pressure $u_{\mathrm{w}}$, Bishop's stress takes the peculiar following form:

$$
\sigma_{i j}^{\prime}=\left(\sigma_{i j}-u_{\mathrm{a}} \delta_{i j}\right)+\chi\left(u_{\mathrm{a}}-u_{\mathrm{w}}\right) \delta_{i j}
$$

where $\chi$ is called the effective stress parameter or Bishop's parameter. Terms $\sigma_{i j}-u_{\mathrm{a}} \delta_{i j}=\sigma_{\text {net }-i j}$ and $u_{\mathrm{a}}-u_{\mathrm{w}}=s$, respectively, define the net stress and the matric suction.

It is recalled that formulation (10) is aimed at averaging the stresses over a representative elementary volume containing all constituents: air, water and solid grains. As mentioned previously, the effective stress should be only representative of solid skeleton stress. A question studied later is the validity of the use of the effective stress as a unique state parameter for the whole partially saturated material. Still, this is verified in the particular case of saturated conditions and incompressible grains and wetting fluid, acting with a neutral stress.

The importance of the effective stress parameter $\chi$ (Equation (10)) lies in the width of range of saturation state it confers to the effective stress. Indeed, the parameter is imposed to vary from 0 
for dry soils to 1 for saturated soils, enabling a simple transition from partially to fully saturated states, and recovering Terzaghi's expression (4) for the saturated case. As a part of the macroscopic expression of stress in the unsaturated medium, the Bishop's parameter was basically introduced to scale down the influence of suction in the function of the volumetric ratios of the different fluid phases. This obviously defines a primary hydro-mechanical coupling by making the effective stress dependent on the matric suction multiplied by a peculiar parameter:

$$
\sigma_{i j}^{\prime}=\sigma_{\text {net }-i j}+\chi s \delta_{i j}
$$

The effective stress parameter has been thought to be likely linked with volumetric fraction of fluids occupying the pore space. Moreover, it is stated that $\chi$ can be different for shear strength or volumetric deformation. Bishop and Donald [11] first attempted to verify Bishop's relation experimentally, and assuming the validity of the Equation (11) in elastic conditions, the values of $\chi$ could be evaluated as follows (Figure 3):

$$
\chi=\tilde{\chi}\left(S_{\mathrm{r}}\right)
$$

One experimental measurement technique of $\chi$ was proposed by Jennings [13] by comparing the behaviour of a soil specimen under changes in applied suction with the behaviour of an identical saturated sample under changes in external pressure. This process lies on the tacit assumption of the validity of the effective stress principle over the whole range of partial saturation, and moreover to verify that all induced deformations from a definite initial state remain in the reversible domain only. Bishop et al. [14] also measured $\chi$ for several soils using volume change and shear strength processes. Zerhouni [12] updated the recapitulative Figure 3 initially taken from [15].

Figure 3 evidences a seemingly trend for $\chi$ to follow the variations of the degree of saturation. Consequently, the uniqueness of relationships between $\chi$ and $S_{\mathrm{r}}$ has been often questioned. A possible elementary choice is written by Schrefler [16] under the following identity:

$$
\chi=S_{\mathrm{r}}
$$

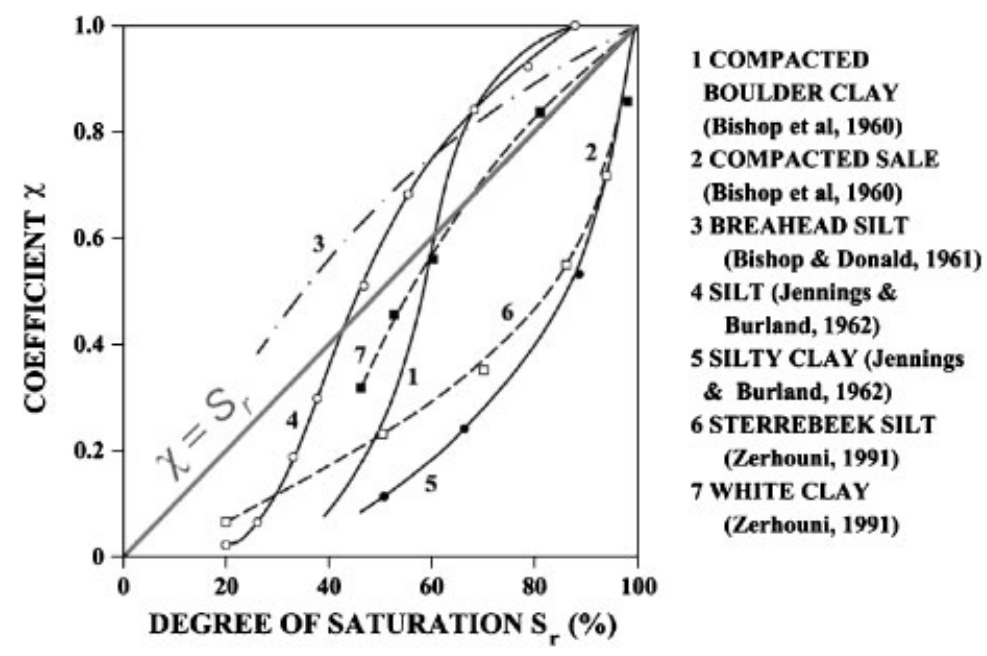

Figure 3. Effective stress parameter versus degree of saturation for a number of different soils in [12]. 
which along with Equation (10) gives the definition of the Bishop's generalized effective stress, or average soil skeleton stress [17]. Detailed framework around this generalized stress will be provided in Section 4.3 showing that this stress is at the basis of numerous recent constitutive stress frameworks. However, early works hardly used this formulation and favoured more complex expressions for parameter $\chi$.

Authors argued that, judging on Figure 3, Equation (13) failed for given ranges of saturation out of $20-80 \%$, that is, for very dry or very wet soils. Aitchison [18] gives thus a fitted expression for the effective parameter, written as follows:

$$
\chi= \begin{cases}1 & \text { if } S_{\mathrm{r}}=1 \\ (\alpha / s) s_{\mathrm{e}} & \text { if } S_{\mathrm{r}}<1\end{cases}
$$

with $s_{\mathrm{e}}$ designating the air entry suction and $\alpha$ a coefficient varying from 0.3 to 0.35 .

Jennings and Burland [15] state in addition that whatever the relationship chosen for parameter $\chi$, there is no unique relationship between volumetric strain and effective stress as defined by Equation (10), and that above a certain critical degree of saturation, the effective stress principle is not valid.

In another recent viewpoint [19], authors recalled that, in reference to [20], parameter $\chi$ might also be related to the current stress and stress history, which, according to the authors, could explain the possibly bad correlation between $\chi$ and $S_{\mathrm{r}}$, which is a volumetric parameter. By plotting $\chi$ against the ratio of matric suction over the air entry value (suction ratio), authors obtain a unique relationship for most soils. Khalili and Khabbaz [21] thus determined a mathematical expression for $\chi$ as a function of matric suction and air entry suction $s_{\mathrm{e}}$ :

$$
\chi= \begin{cases}\left(\frac{s}{s_{\mathrm{e}}}\right)^{-0.55} & \text { if } s>s_{\mathrm{e}} \\ 1 & \text { if } s \leqslant s_{\mathrm{e}}\end{cases}
$$

The advanced framework developed in [21] remains, however, different from the early simple effective stress conceptions previously described. In order to avoid confusion, no more reference to these works will be made until Section 4.3.

4.1.2. Apparent limitations of the single effective stress. Prior to the $1990 \mathrm{~s}$, the effective stress principle extended to partially saturated soils remained basically associated with a fully elastic (linear or not) conception. This elementary approach brings obviously a number of difficulties for constitutive modelling and misleads numerous research teams on the validity of the effective stress. In the following are synthesized the statements apparently arguing in disfavour of the effective stress framework, followed by a new interpretation in the light of the introduction of the elasto-plasticity concepts.

Jennings and Burland [15] were among the first ones to question the validity of Bishop's relation, considering that it could not seem to provide explanation for collapse phenomenon in unsaturated soils. The range of soils of interest here are the so-called heaving and collapsing soils. Collapse phenomenon or plastic compression is observed along a wetting path under a significant constant mechanical load $[15,22]$. Along the same wetting path, reversible swelling can be observed prior to plastic collapse (Figure 4). 


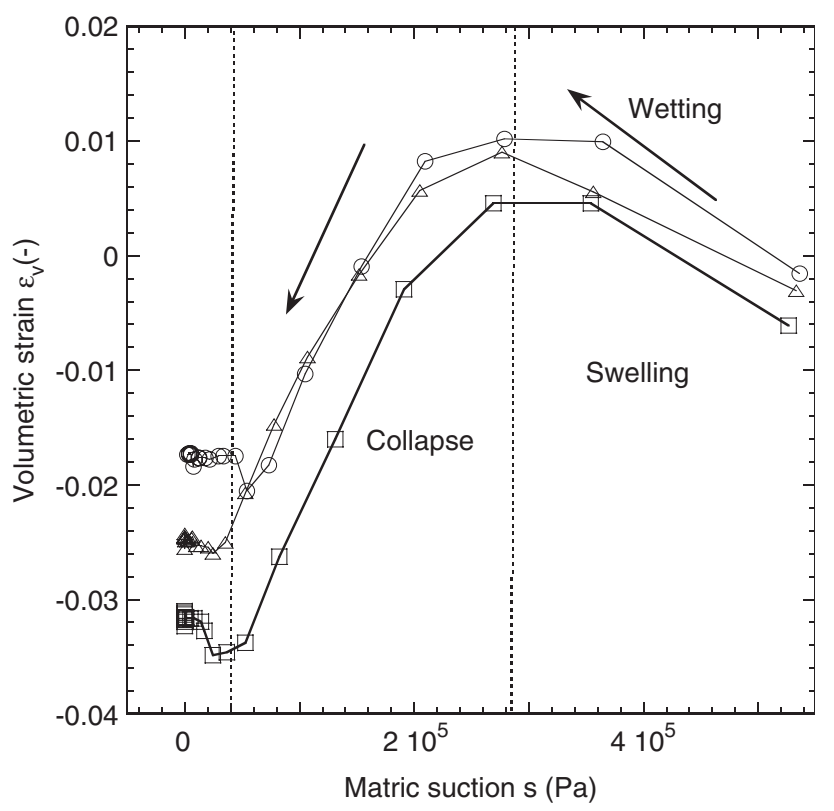

Figure 4. Swelling collapse phenomenon in Kaolin, under a mean net stress of $40 \mathrm{kPa}$. Suction decrease is applied (after [23]).

Within the previously defined limited elastic context, during wetting under constant net stress, the matric suction $s$ decreases down to zero, which in consequence provokes a decrease in effective stress (Equation (10)). This stress reduction should, by definition of a saturated material, induce a slight increment in void ratio, which is contrary to the experimental observation (Figure 4). So the Bishop's generalized effective stress apparently failed to capture the collapsible behaviour of soil, which supported afterwards the determination of other stress state variables as developed in depth in Section 4.2.

Nevertheless, later interpretation of experimental results showed that the collapse phenomenon is linked to plastic behavioural mechanism. In the early 1962s, Leonards [24] had already suggested to interpret the observed compression upon wetting as a sliding of particles with respect to each other, even in all-round compression tests. That rearrangement actually characterizes a plastic phenomenon. These observations show that the arguments against the effective stress were misleading, in the sense that they imputed the limitations to the stress itself instead of judging the inadequacy of the whole reversible context. In the end, it encouraged further use of the effective stress principle associated with complete elastic-plastic frameworks. In addition, the evolution of unsaturated soils knowledge then gave birth to modified effective stresses (e.g. incorporating the effects of phases interfaces) used in an elasto-plastic framework. These considerations are the objectives of Section 4.3.

Another apparent shortcoming of the single effective stress approach is the definition and use of the effective stress parameter, see Section 4.1.1. Provided that the ratio $\chi / S_{\mathrm{r}}$ appeared not to be equal to 1 , the possible difficulties to determine parameter $\chi$ were pointed out. Mainly, the parameter determination is likely to require nonconventional experimental procedures. Once 
more, these arguments should be handled with care, considering that the early effective parameter determination and thus the pseudo-validity of effective stress principle were examined in a fully elastic context. On the other hand, Fredlund and Morgenstern [25] also argue that the effective stress incorporates a soil parameter characteristic, rendering the equation a constitutive expression instead of a mere description of the stress state, which means that supplementary stress variables could be added to get rid of the material parameter.

Other effective stress determination strategies are based on a micromechanical equilibrium analysis. Lu and Likos [5] propose a micromechanical conceptualization of the effective stress. The force equilibrium of a two-soil grain system leads to the identification of an interparticle force balancing the external force, the forces arising from van der Waals attraction, electrical doublelayer repulsion, chemical cementation and lastly the capillary forces. In an opposite viewpoint, Tarantino and Mongiovì [26] affirm that being a macroscopic concept, the effective stress principle could be proved or disproved only on the basis of experimental evidence and not on theoretical models of a microscopic level.

\subsection{Independent stress variables}

4.2.1. Identification of state variables for unsaturated soils. Considering any constitutive model with Bishop's generalized stress seemed unable to describe all features of behaviour of unsaturated soils in a simple form; it has therefore been proposed to adopt a multiple stress variable approach. In immediate response to Jennings and Burland [15], breaking down the effective stress for unsaturated soils, Coleman [20] suggests to write the soil volumetric strain in the following modified manner, under triaxial conditions:

$$
-\frac{\mathrm{d} V}{V}=-C_{21}\left(\mathrm{~d} u_{\mathrm{w}}-\mathrm{d} u_{\mathrm{a}}\right)+C_{22}\left(\mathrm{~d}\left(\frac{1}{3}\left(\sigma_{1}+2 \sigma_{3}\right)\right)-\mathrm{d} u_{\mathrm{a}}\right)+C_{23}\left(\mathrm{~d} \sigma_{1}-\mathrm{d} \sigma_{3}\right)
$$

with $V$ being the overall volume, $\sigma_{1}$ the axial total stress, $\sigma_{3}$ the lateral total stress, and each independent parameter $C_{21}, C_{22}, C_{23}$ related to the material characteristics. Merging the second and third term on the right-hand side of Equation (16) into a single one, we write the constitutive equation as follows, considering small strain hypothesis:

$$
\dot{\varepsilon}_{i j}^{\mathrm{e}}=C_{i j h k}^{\mathrm{e}}\left(\dot{\sigma}_{h k}-\dot{u}_{\mathrm{a}} \delta_{h k}\right)+C^{\mathrm{s}}\left(\dot{u}_{\mathrm{a}}-\dot{u}_{\mathrm{w}}\right) \delta_{h k}
$$

where $C^{\mathrm{s}}$ is termed the elastic hydric modulus (coefficient of proportionality between strain and suction).

Compared with constitutive Equation (1) in Section 2, two independent stress state variables have emerged, resulting in the need for a double constitutive matrix. In that particular case, the stress state is namely described by the net stress and the matric suction. Indeed, the identification of state variables can be based on multiphase continuum mechanics [25], leading to the conclusion that 'any two of the three possible state variables $\left(\sigma ; u_{\mathrm{w}} ; u_{\mathrm{a}}\right)$ can be used to define the stress state', possible combinations being:

$$
\begin{array}{llll}
\text { (1) }\left(\sigma-u_{\mathrm{a}}\right) & \text { and } & \left(u_{\mathrm{a}}-u_{\mathrm{w}}\right), & \text { e.g. used in [27] } \\
\text { (2) }\left(\sigma-u_{\mathrm{w}}\right) & \text { and } & \left(u_{\mathrm{a}}-u_{\mathrm{w}}\right), & \text { e.g. used in [28] } \\
\text { (3) }\left(\sigma-u_{\mathrm{a}}\right) & \text { and } & \left(\sigma-u_{\mathrm{w}}\right) &
\end{array}
$$


Thus, two stresses are proposed for both the soil particles and the contractile skin. The experimental verification of this statement consists in null tests, for which each quantity of the combined stresses is increased by the same amount. In such null tests, there is no tendency for volume change in the overall sample and no change in the degree of saturation. Practically, the verification is possible for the usually controlled stresses, which are the net stress and matric suction. Fredlund and Morgenstern [25] led a number of concluding null tests on silts and kaolin. Tarantino and Mongiovi [26] later corroborated the propositions from Fredlund and Morgenstern, observing neither volume change nor water volume change in null tests practised on kaolin samples. Different apparatuses were used to compare results of a positive air over pressure (axis translation technique) and of a truly negative water pressure via the osmotic technique.

The most frequently used couple of variables in this approach is the combination (1) of Equation (18): the net stress $\left(p_{\text {net }}=\sigma-u_{\mathrm{a}}\right)$ and the matric suction $\left(u_{\mathrm{a}}-u_{\mathrm{w}}\right)$, for physical and practical reasons (e.g. $[22,27])$. The scalar variable $\left(u_{\mathrm{a}}-u_{\mathrm{w}}\right)$, corresponding to suction, has a definite physical meaning, while most of the time the air pressure may be considered constant and equal to atmospheric pressure: $u_{\mathrm{a}}=u_{\mathrm{atm}}(=0)$. Under this assumption, net stress is simplified to total stress and suction becomes a negative water pressure. Moreover, this choice is adapted to the axis translation technique, consisting in the application of $u_{\mathrm{a}}>0$. Using this description, the constitutive law is recalled to be written in the form of Equation (17).

Geiser et al. [28] adopted, however, the second combination of stresses in Equation (18) to overcome problems raised by the use of net stress in describing the whole range of saturation. Further details about these limitations are given later. The original combination of saturated effective stress or Terzaghi's effective stress $\left(\sigma-u_{\mathrm{w}}\right)$ with matric suction $\left(u_{\mathrm{a}}-u_{\mathrm{w}}\right)$ lead to a modified constitutive variational relationship written as follows:

$$
\dot{\varepsilon}_{i j}^{\mathrm{e}}=C_{i j h k}^{\mathrm{e}} \dot{\sigma}_{h k}^{\prime}+C_{\mathrm{s}}^{\prime} \dot{s} \delta_{i j}
$$

with $\dot{\sigma}_{h k}^{\prime}$ being the increment of Terzaghi saturated effective stress, $C^{\prime \mathrm{e}}$ the elastic matrix and $C_{\mathrm{s}}^{\prime}$ an elastic proportionality coefficient for the hydric behaviour, both of which assumed independent each with respect to the other.

Eventually, no matter the combination elected from Equation (18), the use of two independent stress state variables will enable the decomposition of strain increments $\dot{\varepsilon}$ into a mechanical part $\dot{\varepsilon}_{\mathrm{m}}$ and a hydraulic part $\dot{\varepsilon}_{\mathrm{h}}$ :

$$
\dot{\varepsilon}=\dot{\varepsilon}_{\mathrm{m}}+\dot{\varepsilon}_{\mathrm{h}}
$$

Equation (20) is identifiable with both forms of Equations (17) and (19).

4.2.2. Limitations of the independent stress variables approach. Although the initial proposals by Leonards [24] well insisted on the need for considering elasto-plastic mechanisms for behavioural interpretation of unsaturated soils, the first early approach using independent stress variables of Matyas and Radhakrishna [22] still deals only with nonlinear elasticity. The state surfaces describe the volumetric state with respect to net stress state on the one hand and matric suction on the other hand. But what was believed to be a solution to modelling swelling and collapsing behaviour was only valuable along monotonic paths and remained unable to describe, for instance, loadingunloading cycles. The third axis 'matric suction' added to the mechanical stress-strain plane $\left(\varepsilon-\sigma_{\text {net }}\right)$ remained a simple artefact for a better stress-strain curve fitting within a nonlinear fully reversible context. Moreover, the state surfaces have to be determined empirically for each material 
under particular stress state conditions and remain applicable only for strictly similar stress paths. Obviously, the elastic models present the shortcoming of neglecting any irreversible process.

Nevertheless, further models continued to use independent stress variables frameworks, and investigations were dedicated to elasto-plasticity contexts rather than on nature of the stresses. Ever since the preeminent contribution of Alonso et al. [27], most recent models based on the independent stress variables are formulated within a complete elasto-plastic framework, often based on those of the constitutive models for saturated soils. Other weaknesses linked to the nature of stress variables themselves have then to be overcome. In [28], it is considered that the bi-tensorial approach, net stress and suction, could fail to provide a straightforward transition between saturated and unsaturated states, i.e. for a null suction, Terzaghi's effective stress cannot be recovered. In other words, the use of net stress implies that for the saturated state, the constitutive law should be expressed in terms of the total stress instead of the effective stress. To overcome this shortcoming, authors proposed to replace net stress by the 'saturated effective stress' $\left(\sigma-u_{\mathrm{w}}\right)$ and thus enable a natural switching from partially to fully saturated states [28]. However, letting the pore water pressure appear in the mechanical variable may cause the representation of wetting-drying stress paths to be more complex than in conventional net stress and suction planes.

As the models following the independent variable approach were the first ones to deal with elasto-plasticity and coupled yield limits, some fundamental concepts are linked to them, such as the loading collapse (LC), suction increase (SI) and suction decrease (SD) curves. Some of these concepts will be described in Section 5. However, it can be seen that more material characteristics are likely to be determined compared with the effective stress approach (see Equations (17) and (19) where not only the constitutive matrix is needed, but also the hydraulic coefficient of proportionality). Other attempts to use the independent stress variable approach, such as [29] for shear strength of unsaturated soils, base the failure criterion on Mohr-Coulomb criterion. The shear strength is written as follows:

$$
\tau=c^{\prime}+\left(\sigma-u_{\mathrm{a}}\right) \tan \Phi^{\prime}+\left(u_{\mathrm{a}}-u_{\mathrm{w}}\right) \tan \Phi^{\mathrm{b}}
$$

with $\Phi^{\prime}$ being the effective saturated angle of friction, $\Phi^{\mathrm{b}}$ the angle of friction accounting for the matric suction contribution to shear strength and $c^{\prime}$ the effective saturated cohesion. The analysis proved to be complex, considering that the material angle $\Phi^{\mathrm{b}}$ has a nonlinear evolution over wide ranges of suction (Figure 5), meaning that a single determination of $\Phi^{\mathrm{b}}$ could not be representative. Even for more advanced constitutive models (e.g. [27,30]) at least one separate function must be introduced to account for the increase in strength with suction.

Lastly, separating completely the mechanical stress from the hydraulic stress prevents a direct accounting of the hydraulic hysteresis effects on the mechanical stress paths. A deepened discussion on this feature of the hydro-mechanical coupling will be exposed later.

\subsection{Coming back to Bishop-type effective stresses}

Numerous experimental data enable an advanced knowledge of elasto-plastic mechanisms linked to suction-induced phenomena along with the water/air interfaces receding or moving forth.

The average reaction to mechanical external loads has been developed previously and will not be recalled here. Yet, the hydraulic loading, consisting in increasing suction under constant net stress, needs to be clarified. This second type of load, corresponding to drying, could be qualified as internal even though the fluid pressures are experimentally controlled from outside the sample. The mere effect of suction change is double: it modifies directly the global stress state of the 


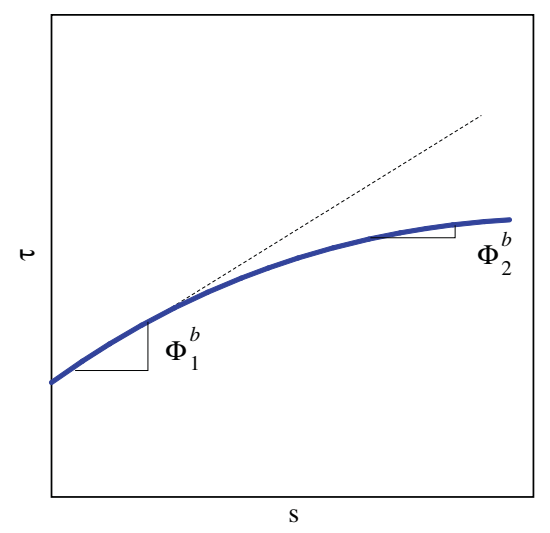

Figure 5. Nonlinearity of apparent friction angle with suction.

material and the saturation being changed, the interfaces' action on the mechanical behaviour does vary. These bonding effects are termed suction-hardening.

From a standard elastic-plastic interpretation and on the basis of experimental observations [31] it is recalled that the latter effects are at the origin of the increase in preconsolidation pressure with suction, and under certain stress conditions, they are linked to the well-known wetting collapse.

Starting form this acquaintance of an inner hydro-mechanical coupling for the unsaturated behaviour, researchers focused back on unified forms of effective stress of the Bishop type, combined with other stress variables to build a proper exhaustive stress framework. In other words, state variables identified by Fredlund and Morgenstern could be combined in different ways to form the effective stress $\sigma^{\prime}$ and completed with a second stress $\xi$. Most of the stress frameworks for the recent models are classified under this family of combined stress. So, in a similar manner to Gens [9] it is proposed to sort the combined stress in two categories according to the level of complexity in the Bishop's like stress formulation and second stress variables.

The first category $(\mathrm{C} 1)$ involves the following couple of stress variables: ${ }^{\ddagger}$

$$
\begin{aligned}
\sigma^{\prime} & =\sigma_{\text {net }}+\tilde{\mu}_{1}(s) \\
\xi & =\tilde{\xi}\left(s, S_{\mathrm{r}}\right)
\end{aligned}
$$

The second category $(\mathrm{C} 2)$ gathers the following type of stress framework:

$$
\begin{aligned}
\sigma^{\prime} & =\sigma_{\text {net }}+\tilde{\mu}_{2}\left(s, S_{\mathrm{r}}\right) \\
\xi & =\tilde{\xi}\left(s, S_{\mathrm{r}}\right)
\end{aligned}
$$

where $\mu_{1}$ and $\mu_{2}$ are functions of suction and/or degree of saturation.

\footnotetext{
¥The convention for notations is the following: a function $f$ depending on variables $x, y$ and $z$ is written under one of the forms $f$ or $\tilde{f}(x, y, z)$. In any other case, the parentheses are used to designate a multiplication. 
4.3.1. Justifications for effective stress type frameworks. The work input characterization within the energetic approaches is a plausible way to identify the possible conjugations of stress and strain variables for constitutive modelling. Observing that in all constitutive frameworks using the net stress and suction as stress state variables, the net stress is implicitly assumed to be work conjugate to the strains; Houlsby [32] proposes to identify the strain-like quantity, that is, work conjugate with suction. Indeed, Houlsby demonstrates that the choice of stress and strain variables is arbitrary, provided that the variables adopted are work conjugate. The rate of input work (per unit volume) to the soil, $\dot{W}$, is expressed as the sum of the products of the stresses with their corresponding strain rates:

$$
\dot{W}=u_{\mathrm{a}} n\left(1-S_{\mathrm{r}}\right) \frac{\dot{\rho}_{\mathrm{a}}}{\rho_{\mathrm{a}}}-\left(u_{\mathrm{a}}-u_{\mathrm{w}}\right) n \dot{S}_{\mathrm{r}}+\left[\sigma_{h k}-\left(S_{\mathrm{r}} u_{\mathrm{w}}+\left(1-S_{\mathrm{r}}\right) u_{\mathrm{a}}\right) \delta_{h k}\right] \dot{\varepsilon}_{h k}
$$

$\rho_{\mathrm{a}}$ being the air density and $n$ the soil skeleton porosity. The three terms of Equation (26) describe the rate of input work to compress the air phase, the rate of input work to change degree of saturation, $S_{\mathrm{r}}$, and the rate of input work to modify the average deformation, respectively. A doubtful assumption in the derivation of Equation (26) is the neglecting of the work dissipated by the air-water interface, the relative velocity between the soil skeleton and the interface supposed to be null. However, other theoretical studies developed later seem to corroborate Houlsby's conclusions.

The power input to compress the air phase assumed negligible, the following simplified relationship is obtained:

$$
\dot{W}=-\left(u_{\mathrm{a}}-u_{\mathrm{w}}\right) n \dot{S}_{\mathrm{r}}+\left[\sigma_{h k}-\left(S_{\mathrm{r}} u_{\mathrm{w}}+\left(1-S_{\mathrm{r}}\right) u_{\mathrm{a}}\right) \delta_{h k}\right] \dot{\varepsilon}_{h k}
$$

The right-hand side terms of Equation (27) could possibly be rearranged in several manners, for instance:

$$
\dot{W}=\left[\sigma_{h k}-u_{\mathrm{a}} \delta_{h k}\right] \dot{\varepsilon}_{h k}+\left[\left(u_{\mathrm{a}}-u_{\mathrm{w}}\right) \delta_{h k}\right]\left(-n \dot{S}_{\mathrm{r}}+S_{\mathrm{r}} \dot{\varepsilon}_{h k}\right)
$$

That means that several combinations of stress-strain conjugates are consistent. For instance, if the net stress is associated with soil skeleton strain (Equation (28)), the generalized strain quantity $\left(-n \dot{S}_{\mathrm{r}}+S_{\mathrm{r}} \varepsilon_{k k}\right)$ must be associated with suction. Equation (27) presents another appealing combination, associating the Bishop generalized effective stress $\sigma^{\prime}=\sigma_{h k}-u_{\mathrm{a}}+S_{\mathrm{r}}\left(u_{\mathrm{a}}-u_{\mathrm{w}}\right) \delta_{h k}$ with the increment of skeleton strain and the matric suction $s=\left(u_{\mathrm{a}}-u_{\mathrm{w}}\right)$ with the product $-n \dot{S}_{\mathrm{r}}$.

Considering that the full implications of Houlsby's assumptions for deriving Equation (26) remained to be investigated, another approach to identify the conjugate stress and strain variables was introduced in [33]. Full demonstrations are also featured in [34]. The authors assume that the soil skeleton is constituted by solid particles and phase interfaces. This point of view is interpretable as a need for taking into account the bonding effects in addition to the identification of the mean volumetric stress acting on the solid particles. Thus, in the case of pore space filled with two fluids all fluid-fluid interfaces and solid-fluid interfaces possess their own proper interfacial energy and entropy and are assumed to act directly on the skeleton free energy $\Psi_{\mathrm{S}}$ :

$$
\Psi_{\mathrm{S}}\left(\varepsilon_{i j}, S_{\mathrm{r}}, T\right)=\psi_{\mathrm{S}}\left(\varepsilon_{i j}, T\right)+n U\left(S_{\mathrm{r}}, T\right)
$$

$\psi_{\mathrm{S}}$ is the free energy of solid matrix per unit of volume, $U$ is the overall interfacial energy per unit volume, $n$ is the porosity and $T$ designates temperature. 
The skeleton state equations of unsaturated thermoporoelasticity are written as follows:

$$
\begin{aligned}
\sigma_{i j}+\pi_{i j} & =\left(\frac{\partial \psi_{\mathrm{S}}}{\partial \varepsilon_{i j}}\right)_{T} \\
\mathbf{S}_{\mathrm{S}} & =-\left(\frac{\partial \psi_{\mathrm{S}}}{\partial T}\right)_{\varepsilon_{i j}} \\
n s & =-\frac{\partial \Psi_{\mathrm{S}}}{\partial S_{\mathrm{r}}}
\end{aligned}
$$

where $\pi_{i j}$, called the equivalent pore pressure, is a function of fluid pressures, degree of saturation and interfacial energy. $\mathbf{S}_{\mathrm{S}}$ is the entropy.

Consequently, a unique stress variable $\sigma_{i j}^{\prime}=\sigma_{i j}+\pi_{i j}$ is sufficient to govern the deformation of the solid skeleton. This effective stress is similar to Bishop's generalized effective stress (see Equations (10) and (13)) to which is added a term accounting for the energy of interfaces. In addition, a second stress variable is defined under the form of suction scaled by porosity; it governs the strain-like quantity called degree of saturation. In the limit state of full saturation, Terzaghi's expression is naturally recovered with the effective stress used in this framework.

In addition, based on a thermodynamic mixture theory, Hutter et al. [35] addressed the conditions allowing the derivation of the effective stress. Following Laloui et al. [36], it is shown that Bishop's generalized effective stress could be thermodynamically consistent if: (i) the soil comprises densitypreserving constituents, (ii) the water is a perfect fluid and (iii) no shear stress is introduced for the fluids. Other thermodynamic analyses of multiphase deformation are exposed in $[37,38]$.

4.3.2. Effective stresses of category 1. It is recalled that the effective stress framework built in the fashion of category 1 set up two stress variables, the effective stress (Equation (22)), which is a particular form of Bishop' s expression, and a second stress variable (see Equation (23)).

In this section, it is proposed to review the main stress frameworks in this category used for constitutive modelling as well as their major advantages and shortcomings. Most of the category 1 effective stress frameworks were defined prior to energetic or thermodynamic approaches mentioned in Section 4.3.1. Consequently, justifications for stress frameworks are often of other particular nature.

Kohgo et al. [39] first defined an effective stress classified in category 1, under the form

$$
\sigma^{\prime}=\sigma-u_{\mathrm{eq}}
$$

where $u_{\text {eq }}$ is called the equivalent pore pressure. This pressure is aimed at averaging the effects of all fluid pressures within the pores. It is also designed to recover Terzaghi's effective stress on saturated states. Consequently, authors had to express the equivalent pore pressure in terms of the air entry suction value, $s_{\mathrm{e}}$, a critical suction, $s_{\mathrm{c}}$, and a material parameter, $a_{\mathrm{e}}$ :

$$
\begin{aligned}
& u_{\mathrm{eq}}=u_{\mathrm{a}}-s \quad \text { if } s \leqslant s_{\mathrm{e}} \\
& u_{\mathrm{eq}}=u_{\mathrm{a}}-\left(s_{\mathrm{e}}+\frac{s_{c}-s_{\mathrm{e}}}{\left(s-s_{\mathrm{e}}\right)+a_{\mathrm{e}}}\left(s-s_{\mathrm{e}}\right)\right) \quad \text { if } s>s_{\mathrm{e}}
\end{aligned}
$$


This formulation is the Bishop's effective stress (Equation (10)) with $\chi=a_{\mathrm{e}}\left(s_{c}-s_{\mathrm{e}}\right) /\left(s-s_{\mathrm{e}}+a_{\mathrm{e}}\right)^{2}$ for unsaturated states and $\chi=1$ for saturated range. The empirical relationship has been formulated on the basis of the relationship between shear strength of unsaturated soil and amount of suction. Out of the promising elasto-plastic considerations, authors define a second stress variable $\xi=s^{*}=s-s_{\mathrm{e}}$ named effective suction to complete the stress framework. This emergence of a second stress variable in the context of effective stress is a pioneering formulation that could be later confirmed by the energetic approach.

Modaressi and Abou-Bekr [40] basically focused on one of the features presented above, that is, the shear strength increasing and reaching a limit upon SI. Then, the proposed effective stress for unsaturated states is simply written as follows:

$$
\sigma^{\prime}=\sigma+\tilde{\pi}_{\mathrm{c}}(s)
$$

$\pi_{\mathrm{c}}$ is named the capillary pressure originally determined by Taibi [41], defined as a hyperbolic function of suction varying from the air entry suction to a certain maximum value linked to the void ratio, surface tension and granulometry of the modelled material. The capillary pressure is quantified by the means of an averaging process of capillary stresses. Other particularity lies in the range of applicability of the capillary pressure; actually, the true form of the effective stress is

$$
\sigma^{\prime}=\sigma+u
$$

with a condition expressed on air entry suction $s_{\mathrm{e}}$ to recover Terzaghi stress for saturated states:

$$
u=-u_{\mathrm{w}} \text { if } u_{\mathrm{w}} \geqslant s_{\mathrm{e}} \quad \text { or } \quad u=-\pi_{\mathrm{c}} \text { if } u_{\mathrm{w}}<s_{\mathrm{e}}
$$

While it is clearly stated by authors that the generalized effective stress concept is applied and, thus, that the given effective stress alone governs the skeleton deformation, the energetic consistency can be verified only if a second set of work-conjugate stress and strain variables is defined in parallel. This second stress $\xi$ and strain are very likely to be expressible in terms of matric suction, porosity and degree of saturation.

Already cited previously is the constitutive framework from Khalili and Khabbaz [21], in which the effective stress is very close to those previously mentioned. The following Equation (37) is exactly Bishop's expression, with a particular function of suction for the effective stress parameter $\chi=\tilde{\chi}(s)$

$$
\sigma_{i j}^{\prime}=\sigma_{\text {net }-i j}+\tilde{\chi}(s) \times s
$$

Once more, the function of suction is defined differently whether the air entry suction is exceeded or not, see Equation (15) in Section 4.1.1, and a material parameter is introduced in $\tilde{\chi}(s)$. This form of effective stress coefficient was determined on the basis of examination of shear strength and volume change data. Again, similar to other models of category 1, no description of a second stress-strain couple is provided although it seems to be necessary at least to describe the hydraulic behaviour in parallel.

To conclude, several forms for function $\tilde{\mu}_{1}(s)$ in Equation (22) have been defined and present similar characteristics, specially giving importance to the air entry suction and involving material parameters. However, since no information on the degree of saturation is required to fully describe $\tilde{\mu}_{1}(s)$, most authors seem to have left apart the second couple of work-conjugate stress and strains, which make the hydro-mechanical behaviour incomplete. Moreover, category 1 effective 
stresses, due to their inner complexity, might lead to a difficult representation in the conventional (experimental) stress-strain planes. Lastly, similar to the independent stresses approach, category 1 effective stresses do not offer a direct unified way to incorporate hydraulic hysteresis effects, as explained later.

4.3.3. Effective stresses of category 2. Category 2 gathers most of the stress frameworks for advanced modelling of unsaturated soils during the last five years. Again, the skeleton deformations are fully governed by a single effective stress of the Bishop type, to which a second stress variable is added, often to complete the description of the hydraulic behaviour. The set of stresses takes the form of Equations (24) and (25).

It has been shown previously that several forms are consistent for functions $\tilde{\mu}_{2}\left(s, S_{\mathrm{r}}\right)$ and $\tilde{\xi}\left(s, S_{\mathrm{r}}\right)$. Consequently, the choices made out by authors are a matter of convenience, provided that in any case the bonding effects and the irreversible processes ought to be accounted for in addition. Most research works tend, however, towards simple stress formulations inside more complex elastic-plastic contexts.

Even though the original definition of the Bishop's generalized effective stress is due to Schrefler [16], one of the first constitutive frameworks making use of this unified effective stress is that of Jommi and Di Prisco [42] later reported in [17]. Based on the stress identification of Houlsby's remarks of work input, the effective stress called average skeleton stress by the authors is defined as the difference between the total stress and the mean value of the fluid pressure weighted with the saturation degree $S_{\mathrm{r}}$ :

$$
\sigma^{\prime}=\sigma_{\text {net }}+S_{\mathrm{r}} s
$$

As pointed out by the authors, the formulation includes an implicit direct dependency of the overall behaviour on the hydraulic state, reflected through variables suction and degree of saturation. In addition, the knowledge of the soil water retention curve is necessary, both to complete the stress framework by monitoring a second set of stress and strain variables and to determine the generalized effective stress itself anytime (Equation (38)).

Several similar stress frameworks are reported in literature (e.g. [43]) but noticeable differences then appear in choice of complementary stress variable $\xi$, Equation (25). Due to the simplicity of its formulation, the Bishop generalized effective stress alone is not able to include advanced effects of partial saturation. Recalling the statements from introduction of Section 4.3, experimental observation showed that an increase in suction is likely to produce two separate effects.

Firstly, a global deformation is induced. From a constitutive point of view, and without entering elastic-plastic considerations, the straining of the material is governed by changes in the effective stress. The formulation of the generalized effective stress (38) unveils a direct modification of $\sigma^{\prime}$ upon suction change.

Secondly, the suction-induced hardening must be accounted for. These hardening effects are believed to be attributable to bonding/debonding effects due to the evolution of interfaces. It is proposed to gather these effects under the generic term of bonding. Since bonding is not included in Bishop's generalized effective stress, some authors suggested including it into the second stress variables. Others preferred keeping the second stress variable simple and introduce advanced coupling in the whole elasto-plastic model formulation. This modification is often referred to as 'second suction mechanism' [17]. 
For instance, Sheng et al. [44] motivated their choice of the following work-conjugate stresses and strains:

$$
\left(\begin{array}{l}
\sigma^{\prime} \\
s
\end{array}\right) \text { and }\left(\begin{array}{c}
\varepsilon \\
S_{\mathrm{r}}
\end{array}\right)
$$

on judging the possible implementation of their constitutive model in finite element codes. Indeed, the proposed stress framework seems to be the best choice, provided that most existing finite element codes are programmed for saturated soils. Consequently, a natural extension to partially saturated states is provided, by simply exchanging effective stresses with Bishop's generalized stresses. Moreover, the second stress strain relationship represents directly the soil water retention curve, which constitutes a relevant output.

Tamagnini's proposal for stress framework [45] is strictly the same as the one above. To introduce the second suction mechanism, the evolution of the preconsolidation pressure $p_{\mathrm{c}}^{\prime}$ is defined in terms of a double-hardening mechanism, under the following form:

$$
\dot{p}_{\mathrm{c}}^{\prime}=\dot{p}_{\mathrm{c}(\mathrm{SAT})}^{\prime}+\dot{p}_{\mathrm{c}(\mathrm{UNSAT})}^{\prime}
$$

with the second term on the right-hand side depending directly on the degree of saturation and material parameters. The relative simplicity of the stress variables thus makes it necessary to introduce refinements in the formulation of the hydro-mechanical couplings. These are also required for modelling the collapse phenomenon upon wetting.

Wheeler et al. [46] also make use of the work input considerations to justify their choice of the Bishop generalized effective stress and a second stress variable named the modified suction. This stress variable, work conjugate with decrement in the degree of saturation, is the product between matric suction and porosity:

$$
s^{*}=n s=n\left(u_{\mathrm{a}}-u_{\mathrm{w}}\right)
$$

The proposed constitutive model includes remarkable hydro-mechanically coupled mechanisms, enabling to reproduce bonding effects in a unified approach. Such a constitutive context, however, implies the use of multiple coupled yield loci and a high level of complexity in the formulation.

A quite opposite contribution concerning the second stress variable is the work from Gallipoli et al. [47]. The authors consider that many features of the elasto-plastic behaviour are linked to bonding phenomenon between particles, which are linked to menisci. As bonding cannot be accounted for by exclusively the average skeleton stress as a constitutive variable, the variable $\xi$ is introduced as a measure of the magnitude of the interparticular bonding:

$$
\xi=\tilde{f}(s)\left(1-S_{\mathrm{r}}\right)
$$

Factor $\left(1-S_{\mathrm{r}}\right)$ is aimed at accounting for the number of water menisci per unit volume of unit fraction and obviously drops down to zero when the soil is saturated. $\tilde{f}(s)$ is a function of matric suction expressing the ratio between interparticle forces at suction $s$ and at null suction (Figure 6). $\tilde{f}(s)$ is determined via a spherical model of two soil grains.

Other proposals tend to include directly the interfaces effects within the mechanical effective stress, which leads to a different content for function $\tilde{\mu}_{2}\left(s, S_{\mathrm{r}}\right)$ in (24) in comparison with Bishop's generalized effective stress. 


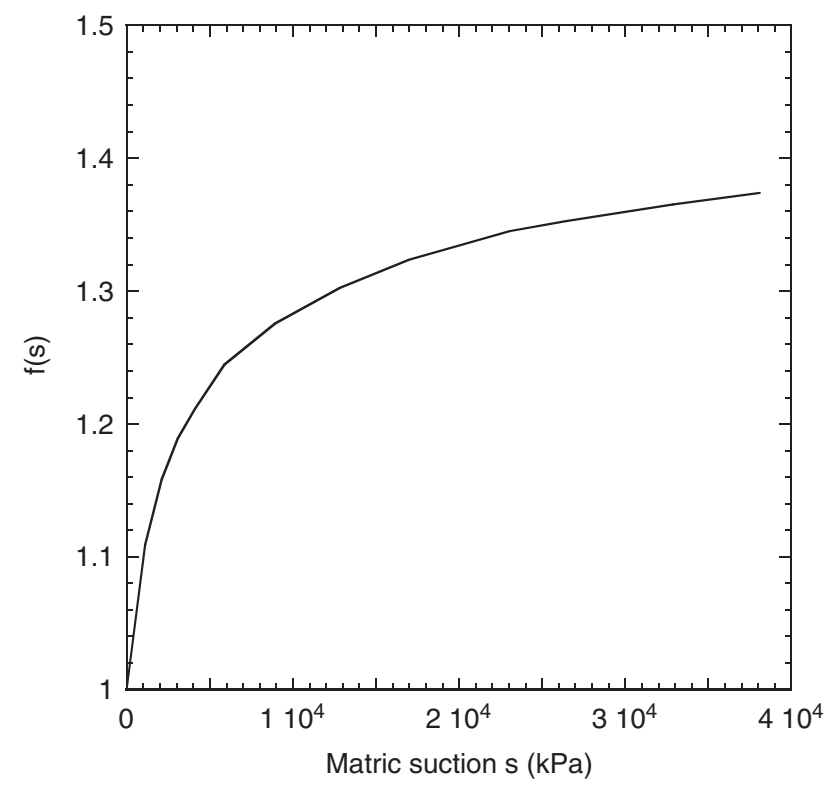

Figure 6. Simplified plot of $f(s)$, from [47].

Studies of interest in this field are Dangla and Coussy [33] followed by Pereira et al. [48]. As demonstrated previously, authors write the effective stress as follows, keeping the notations from Section 4.3.1:

$$
\sigma_{i j}^{\prime}=\sigma_{i j}+\pi_{i j}
$$

with $\pi_{i j}$ being the equivalent pore pressure defined as

$$
\pi_{i j}=\left(u_{\mathrm{a}}-S_{\mathrm{r}}\left(u_{\mathrm{a}}-u_{\mathrm{w}}\right)\right) \delta_{i j}-\left(\frac{\partial(n U)}{\partial \varepsilon_{i j}}\right)_{S_{\mathrm{r}}, T}
$$

The second set of conjugate stress and strain variables can be taken from Equation (32). The advantage of the proposed stress framework is that the energy of interfaces is included into the effective stress, simplifying the modelling of bonding. However, the quantification of the interfaces energy $U$ is based on the knowledge of the water retention curve:

$$
U\left(n, S_{\mathrm{r}}\right)=\int_{S_{\mathrm{r}}}^{1} \tilde{s}(n, S) \mathrm{d} S
$$

A major advantage of this determination is that quantity $U$, with reference to (45), is directly dependent on the soil water retention curve. Consequently, if the drying path and the wetting paths are not confounded in plane $\left(S_{\mathrm{r}}-S\right)$, evidencing a hydraulic hysteresis, the interfaces energy will reflect this irreversibility. In the end, the hysteresis effects are naturally incorporated within the effective stress. 
More generally, this natural accounting for the hydraulic hysteresis in the stress state is valuable for any effective stress belonging to category 2, provided that both the degree of saturation and the matric suction enter its formulation. Basically, if the main wetting curve and main drying curve can be separated, the stress paths in plane $\left(\tilde{\xi}\left(s, S_{\mathrm{r}}\right)-\sigma^{\prime}\right)$ will be invariably different upon drying and wetting. Graphical representation of this feature is provided in Section 5.3.2. This direct incorporation of hydraulic hysteresis within the skeleton stress variable is neither possible for the independent stress variables approach nor in the category 1 of effective stresses.

As a conclusion, the effective stress frameworks classified in category 2 present first the advantage of providing a natural transition between saturated and unsaturated states. The existence of $\tilde{\mu}_{2}\left(s, S_{\mathrm{r}}\right)$ facilitates the inclusion of hydraulic effects, and particularly hydraulic hysteresis. Using an effective stress imposes a higher level of complexity for the stress variable, but potential advantage of expressing a constitutive model in terms of relatively complex stress variables is that the relationships between strain, strength and these stress parameters should be simpler than for a model expressed in terms of net stress and suction, if the stress variables have a strong physical significance. Some authors still argue that the representation of stress paths in conventional stress-strain planes is not straightforward, or almost impossible in the case of extremely sophisticated effective stresses, indicating that if a category 2 effective stress is to be elected, the simple formulations such as Bishop's generalized effective stress should be preferred.

4.3.4. Concluding remarks. While it is now clear that any adequate set of work-conjugate stresses and strains can be used for constitutive modelling of unsaturated soils [9], the difference likely comes from the convenience in manipulating stress-strain planes and secondary functions.

Examining the pros and cons of stresses formulations [9], it has appeared that the effective stresses from category 2 own the highest number of advantages, including the straightforward transition between saturated and unsaturated states and the direct accounting for the hydraulic hysteresis effect on the mechanical response. This framework makes the effective stress principle applicable for describing the mechanical part of the behaviour, resulting in very likely simplified stress-strain relations. In addition, since the determination of the effective stress shall not be fastidious due to high number of specific parameters, the simplification of the function $\tilde{\mu}_{2}\left(s, S_{\mathrm{r}}\right)$ down to the product $\left(s \times S_{\mathrm{r}}\right)$ is a promising option. In the following, the Bishop's generalized effective stress is thus adopted as a standard and broadly recognized effective stress for describing the mechanical behaviour of unsaturated soils. Obviously, the hydraulic part of the behaviour must be described in addition; as the objective of the paper is mostly to validate a mechanical effective stress, the hydraulic stress and strain are taken simple, as written explicitly in the complete stress-strain framework as follows:

$$
\left(\begin{array}{c}
\sigma^{\prime}=\sigma_{\text {net }}+S_{\mathrm{r}} s \\
s=u_{\mathrm{a}}-u_{\mathrm{w}}
\end{array}\right) \quad \text { and } \quad\left(\begin{array}{c}
\varepsilon \\
S_{\mathrm{r}}
\end{array}\right)
$$

The adoption of the previous framework lies on the conceptual comparative analysis of stresses for unsaturated soils. It is proposed to validate this stress selection on the basis of experimental data, by answering two main issues, namely (i) checking the actual simplifications due to the effective stress formulation and (ii) investigating the apparent shortcomings usually attributed to Bishop's generalized effective stress. 


\section{IMPLICATIONS OF THE USE OF A GENERALIZED EFFECTIVE STRESS}

The implications of the use of the Bishop's generalized effective stress for the interpretation of the unsaturated mechanical behaviour are studied in the following. The hereby assertions are exclusively based on experimental data and form the pretext of an advanced constitutive model [49]. The principle is to re-plot experimental data sets expressed in terms of classical unsaturated stress variables, which are net stress and suction into new effective stress planes. The uniqueness of critical state line (CSL) for different suctions and modifications along unsaturated mechanical loading paths are investigated. Other implicit effects of the hydro-mechanical coupling are also presented, leading to introductory discussion on modelling.

For the sake of simplicity, in the following, the conventional triaxial stresses are used for representations. The deviatoric stress $q$ and mean stress $p$ are defined as follows:

$$
\begin{aligned}
& q=\hat{\sigma}_{11}-\hat{\sigma}_{33} \\
& p=\frac{\hat{\sigma}_{11}+\hat{\sigma}_{22}+\hat{\sigma}_{33}}{3}
\end{aligned}
$$

$\hat{\sigma}_{i j}$ being the stress tensor chosen: depending on stress framework, it can be the Bishop's generalized effective stress $\sigma^{\prime}$ or the net stress $\sigma_{\text {net }}$.

\subsection{Critical state analysis}

Khalili et al. [19] investigated the uniqueness of the CSL in a deviator stress versus mean effective stress plane for different levels of suction. The stress variable used for reinterpretation has been given under the form of Equation (11) with the effective stress parameter $\chi$ being a function of suction and air entry value, see Equation (15). Results evidence clearly a convergence of all critical state points towards a single line whatever the suction, as plotted, for instance, in Figure 7.

However, authors do not provide any conventional net stress interpretation plot for comparison with the new effective stress plot, preventing to estimate whether it is really worth or not to use the effective stress and if the conversion is significant. We propose to reinterpret similarly several shearing data sets with the Bishop's generalized effective stress, termed 'effective stress' in the following for the sake of simplicity.

Figures 8 and 9 mirror two stress interpretations for CSL, namely the $\left(q-p_{\text {net }}\right)$ plane (Figures 8(a) and 9(a)) and $\left(q-p^{\prime}\right)$ plane (Figures 8(b) and 9(b)). Most of the unsaturated experimental behavioural studies report data in conventional net stress planes, the latter being an experimentally controlled stress variable. Under this form, even though the variations of the friction angle and cohesion are evidently different from one material to another, Figures 8(a) and 9(a) highlight a plurality of CSLs in the net stress planes for both materials.

Whereas transforming the plot from net stress conception to effective stress representation does not affect deviatoric stress level, the isotropic effect of suction scaled by degree of saturation is added to the net mean stress. The CSLs at different suctions are uniformly translated horizontally by the amount of stress mentioned above, the immediate consequence of which is a reduction of apparent cohesion. The alignment property of points is conserved in the new plane as well as friction angles.

The processed results of Sivakumar [23] in Figure 8(b) are particularly accounting for an obvious unification of the effective critical state whatever the level of suction between 0 and $300 \mathrm{kPa}$, 

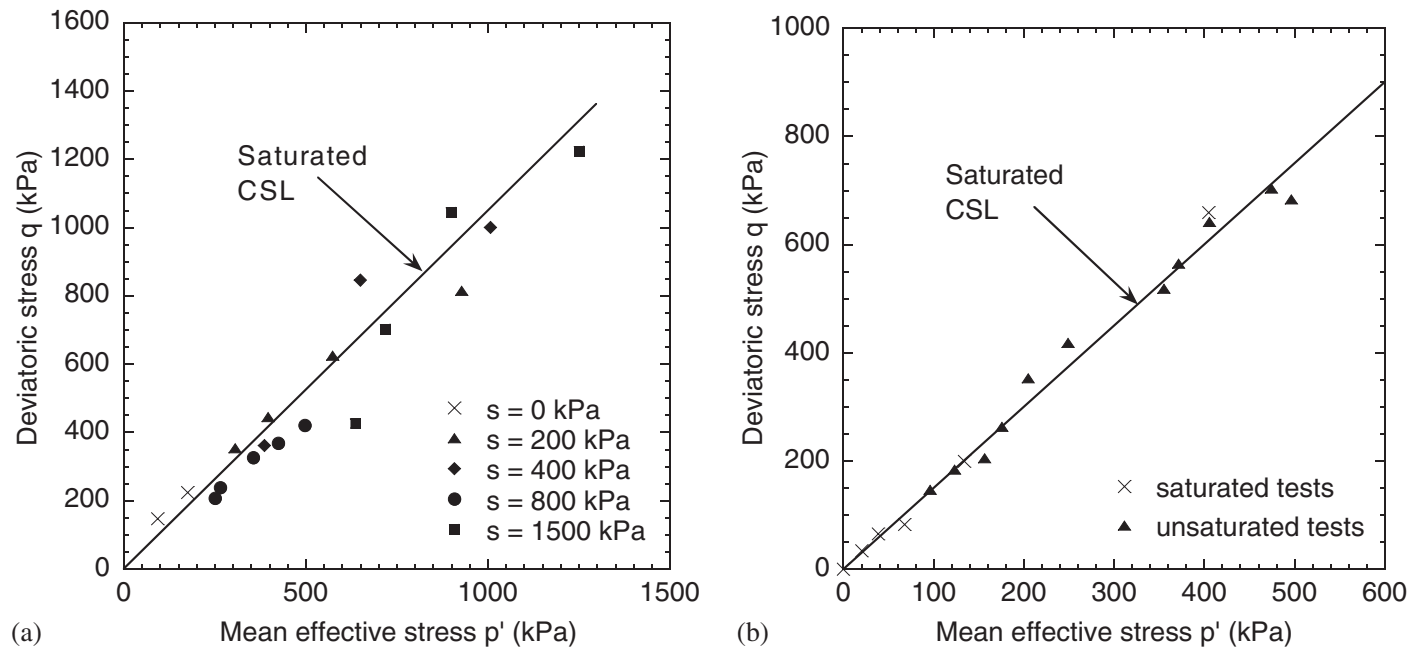

Figure 7. Critical state evolution with suction in $\left(q-p^{\prime}\right)$ plane for: (a) Jossigny silt [50] and (b) trois rivières silt [51], reported by [19].
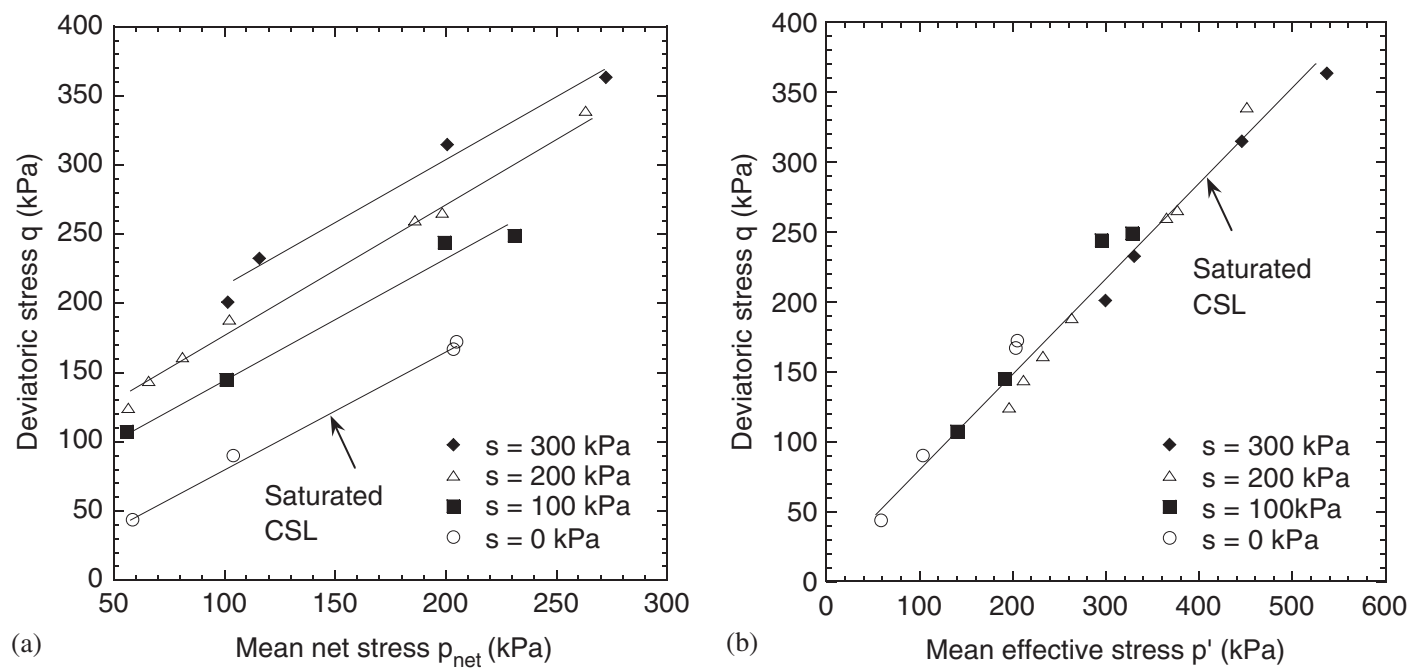

Figure 8. Critical state lines for Kaolin at different suctions-experimental data from [23].

which corresponds to $1 \leqslant S_{\mathrm{r}} \leqslant 0.59$. Concerning Sion silt [31], while the net stress interpretation, Figure 9(a), already evidences a narrow arrangement of lines, the effective stress version, Figure 9(b), tends to align even more the experimental points and sets the whole scattering much closer to the saturated CSL, accounting for its uniqueness.

This encourages simplification of parameter determination, assuming that saturated shear parameters, namely the saturated angle of friction and cohesion, are sufficient to describe both 

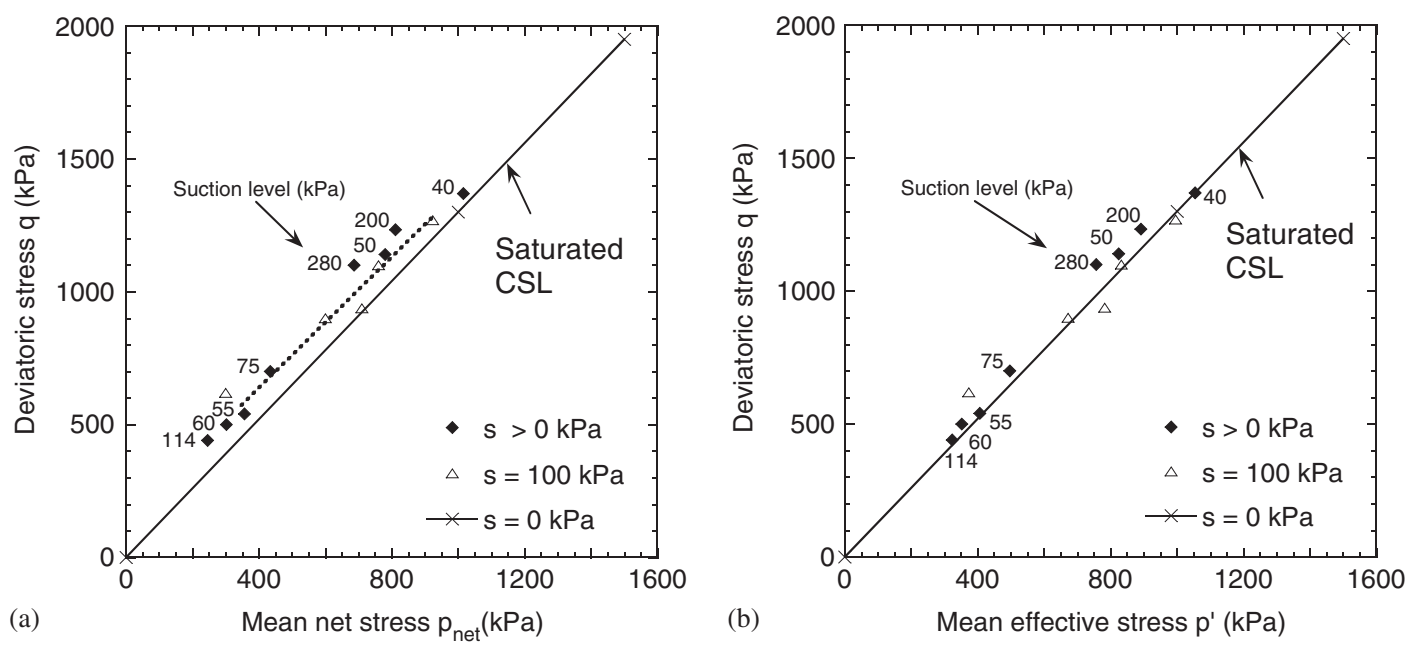

Figure 9. Critical state line for Sion silt at different suctions. Experimental data from [31].

saturated and partially saturated critical state behaviours, overcoming the difficulties linked to the suction-dependent cohesion observed in the $\left(q-p_{\text {net }}\right)$ plane.

\subsection{Unsaturated mechanical compression}

Detractive arguments regarding an effective stress framework concern the apparent difficulty to evaluate the stress level and then to interpret results in subsequent effective planes. However, even if the stress variables are not directly the experimentally controlled ones, which are net stress and suction, the elected generalized effective stress is a rather simple combination of them, with the parallel retention information. Nevertheless, data sets lacking from continuous hydraulic monitoring (that is knowledge of water content, volumetric strain and matric suction) are obviously excluded from the proposed effective stress interpretation.

Figure 10 indicates that the increase in compressibility and preconsolidation pressure with suction, observed in the experimental net stress mechanical planes, Figure 10(a), is recovered in the effective stress interpretation, Figure 10(b). On the whole, those two suction effects appear to be amplified with the proposed generalized effective stress approach.

Gallipoli et al. [47] plotted a similar interpretation with the Bishop's generalized effective stress, interpreting slightly curved shapes for compressions rather than lines, Figure 11. However, Figure 10 shows that a linear model could fit well the compression lines in the plastic part and that inflexions of the experimental curves, if some, are likely to appear only at high mean effective stresses or might not be significant.

Figure 10 also enables the determination of the LC yield curve, the original definition of which is due to Alonso et al. [27] in the matric suction versus mean net stress plane. This yield limit accounting for the increase in preconsolidation pressure $p_{\mathrm{c}}$ with suction is a reversible function that reflects the suction hardening, see Figure 12. As expected from previous observations, the shape of the LC curve in the effective stress interpretation is similar to the reference one, but neatly amplified. A significant consequence is that analogous mathematical formulations for the function $p_{\mathrm{c}}=\hat{p}_{\mathrm{c}}(s)$ could be applied to both net and effective stress analyses. 

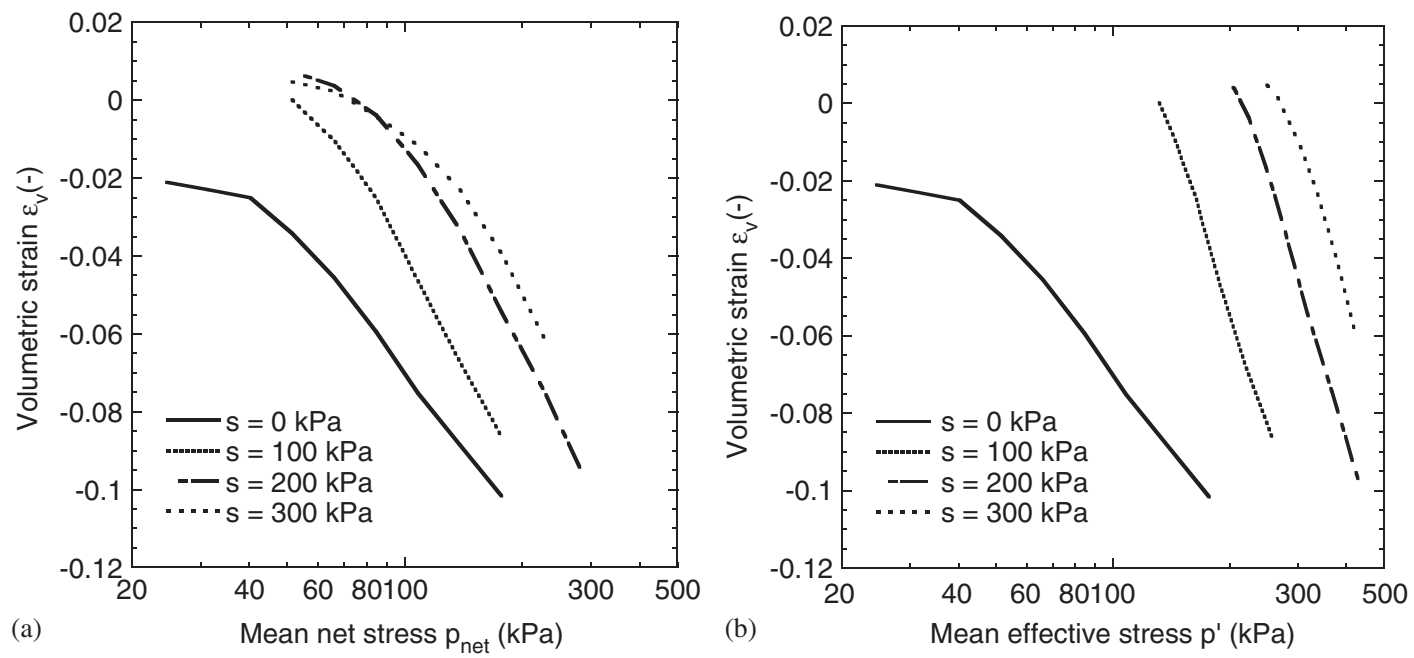

Figure 10. Isotropic compressions of kaolin at different suctions, experimental data from [23].

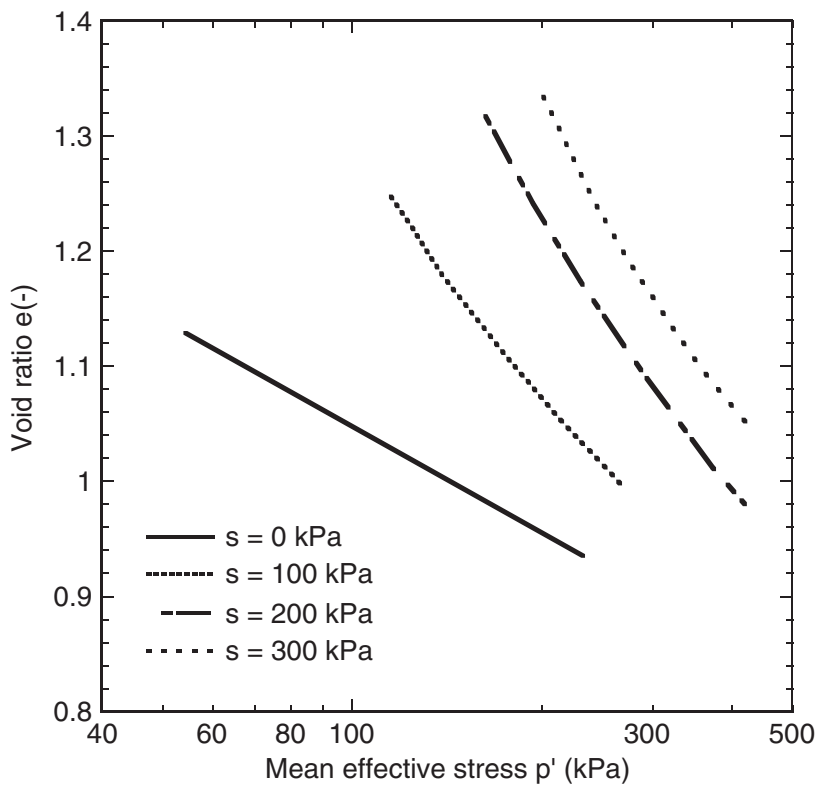

Figure 11. Normal compression lines at constant suction in the plane void ratio versus mean effective stress (experimental data from [23]), after [47].

Concerning the saturated domain, defined by $s<s_{\mathrm{e}}$ with $s_{\mathrm{e}}$ being the air entry suction, the shape of the yield limit is a simple vertical line in the $\left(s-p_{\mathrm{c}}^{\prime}\right)$ plane. The effective stress interpretation induces this independency of the apparent preconsolidation pressure on suction, unlike in the $\left(s-p_{\mathrm{c}-n e t}\right)$ plane. This specificity owns a fundamental use for the modelling of volume changes 


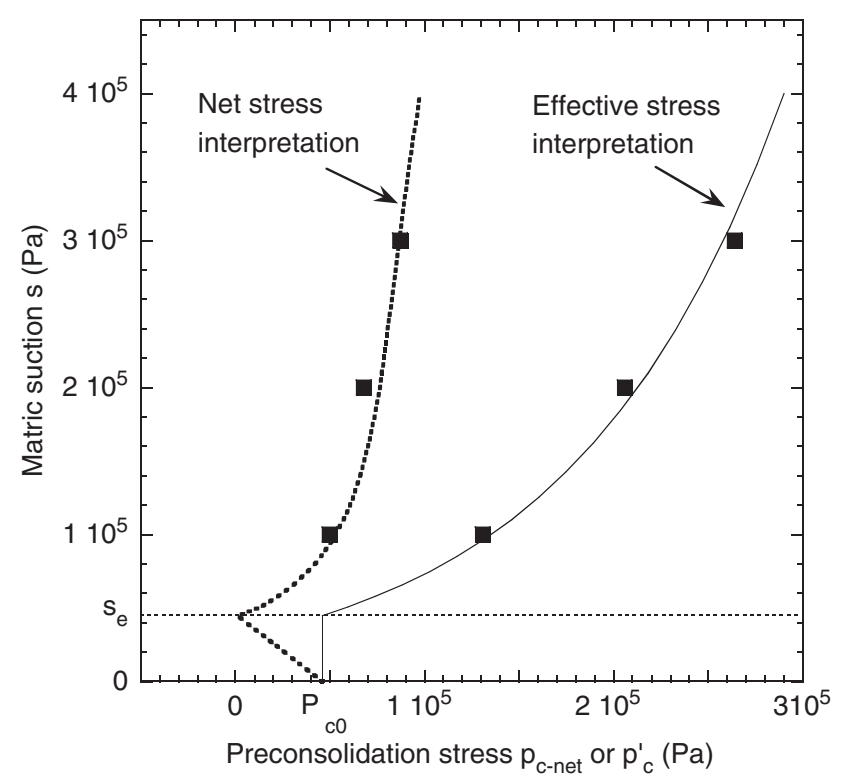

Figure 12. Shape of the LC curve in two interpretations, experimental data from [23].

upon hydraulic loading paths [49]. Notice that the transition between saturated and unsaturated states being not straightforward with the net stress and suction interpretation, it is certainly incorrect to let appear the saturated zone $\left(s \leqslant s_{\mathrm{e}}\right)$ in the $\left(s-p_{\mathrm{c}-\mathrm{net}}\right)$ plane.

\subsection{Constitutive modelling framework}

As elasto-plastic aspects will be introduced in this section, the reader must be aware that the terminology 'generalized' is solely applied to stress variables and has no relation with generalized plasticity concept.

5.3.1. Unicity of yield limit. Statements in Section 2 imply that the effective stress is simply defined as that inducing the mechanical (elastic) strain of the solid skeleton, Equation (1). Judging on Equation (11), the generalized effective stress is incremented by the application of any combination of the external mechanical stress $\sigma_{i j}$ and the matric suction $s$. So, any of the two separate or combined loads induce variations in total volumetric strain $\dot{\varepsilon}_{\mathrm{V}}$ that is equivalent to a pure mechanical straining of the material, as expected from constitutive Equation (1).

On this basis, the mechanical stress state being fully described by means of the unified stress, plasticity mobilization can be evaluated via the generalized stress state alone. A relevant consequence of the unified stress approach lies thus in the sufficiency of a single mechanical yield surface to comprehend any elasto-plastic behaviour resulting in skeleton deformations. So, for the mechanical stress-strain behaviour, no second yield surface in suction is required, setting the model formulation free from any kind of SI or SD yield curves, which are proper to constitutive models written in terms of independent stress variables such as Barcelona Basic Model family [27] or others [52]. 


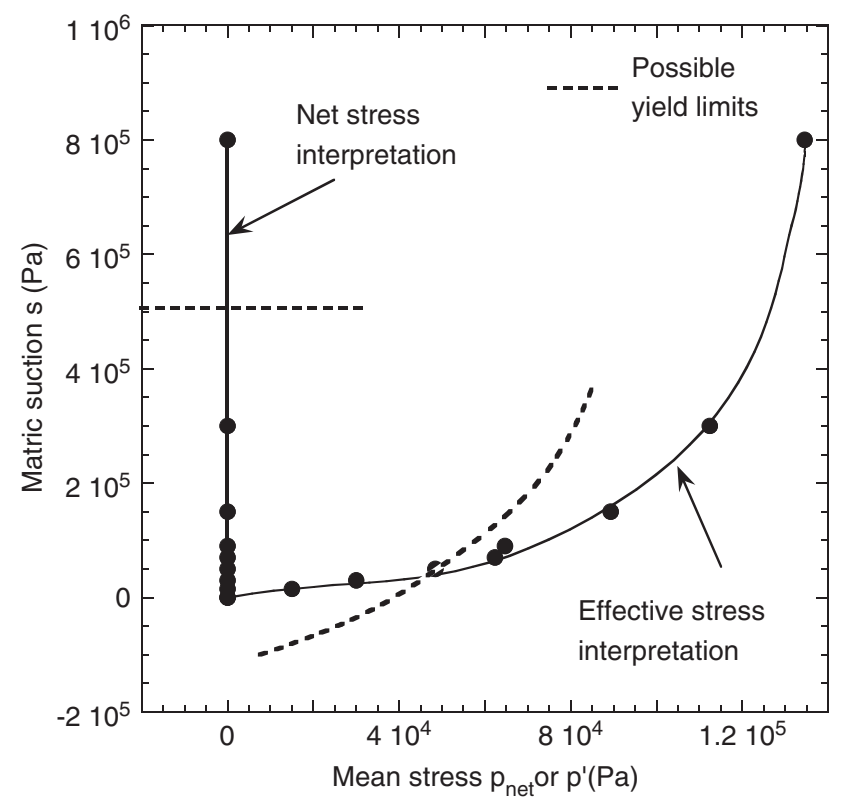

Figure 13. Drying path in two interpretations on Sion silt, experimental data from [31].

In the end, the LC curve should include a hydro-mechanical coupling, which leads to the following formulation:

$$
p_{\mathrm{c}}^{\prime}=\hat{p}_{\mathrm{c}}^{\prime}(\zeta, s) \quad \forall s
$$

where the term $\zeta$ gathers the saturated hardening variables. It can be remarked that the expression for LC yield curve is continuous from the saturated state to the unsaturated state.

Visualizing the stress paths in the matric suction versus mean stresses planes help understanding better the unified stress framework implications, Figure 13. Whereas a drying path under constant net stress is a simple vertical line in the $\left(s-p_{\text {net }}\right)$ plane, the effective stress formulation confers a curved shape in the $\left(s-p^{\prime}\right)$ plane. With reference to Figure 12, the effective drying path evidences a possible direct yielding on the LC curve, whereas the net stress interpretation shows the need for a supplementary pseudo-horizontal yield limit.

A questionable issue remains the fact that the curvature of the drying path in the effective stress conception is narrowly linked to the soil water retention curve shape. Indeed, it has been seen that the product of $s$ times $S_{\mathrm{r}}$ in the formulation of the effective stress confers to it a nonlinear evolution upon suction is changes. But, upon drying, once a certain limit in suction is reached, called $s_{\mathrm{CR}}$ as critical suction, the $S_{\mathrm{r}} s$ product tends to decrease, resulting in an inflexion in the stress path representation (plane $\left(s-p^{\prime}\right)$ ) and a decrease in the effective stress. However, this inconsistency may appear only at very high suctions, where the degree of saturation drops down to its residual value close to zero, see Figure 14. Gray and Schrefler [54], for instance, related this aspect to the residual state of saturation, where water phase is described as a film coating the particles rather than under the form of a connected reservoir. Then, a modified water pressure can be introduced 

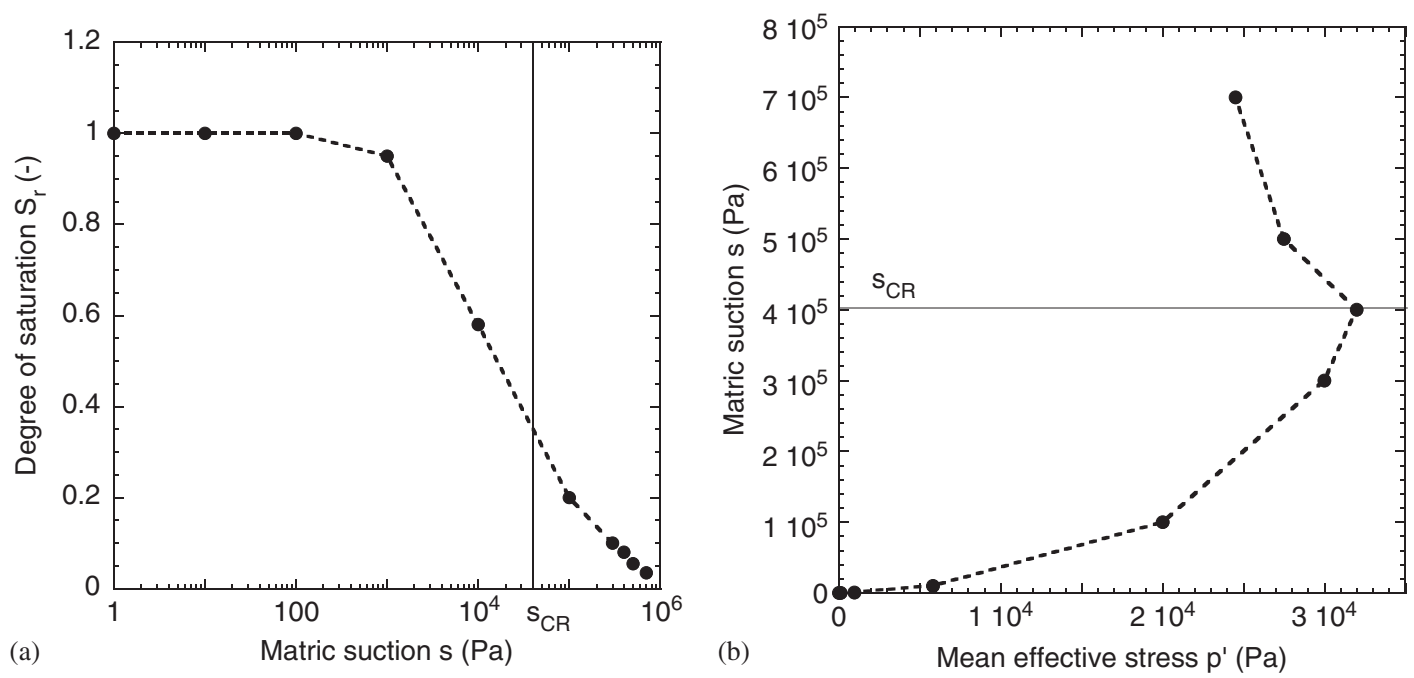

Figure 14. Characterization of the critical suction: (a) soil water retention curve-drying path and (b) corresponding stress path in $\left(s-p^{\prime}\right)$ plane. Experimental data from [53].

to correct the effective stress tensor. Consequently, a particular attention must thus be paid for interpreting this limit state with the generalized effective stress proposed here.

5.3.2. Hydraulic hysteresis. A unique feature conveyed by Bishop's generalized effective stress is taking into account of no less than two effects of the hydro-mechanical coupling. Firstly, as developed previously, any increase in suction is likely to provoke a direct change in the effective stress, Equation (46). Secondly, the defined stress framework enables a natural repercussion of the hydraulic hysteresis observed in $\left(S_{\mathrm{r}}-s\right)$ plane into $\left(s-p^{\prime}\right)$ representation. Figure 15(a) plots a typical shape of a soil water retention curve in the conventional $\left(S_{\mathrm{r}}-s\right)$ plane. A particular attention must be paid to the drying curve being not recovered upon wetting, letting appear a dissipation characterizing the hydraulic hysteresis. The two limiting curves are usually termed main drying curve and main wetting curve. Notice, however, that different intermediary paths are possible within the space bounded by the main wetting and drying curves. Obviously, the consequence of the hydraulic hysteresis is that for a same level of suction, two different states of saturation can be obtained according to the suction variation in progress. Although this has nothing to do directly with suction-induced hardening mentioned previously, or bonding, the stress state and skeleton deformations are all the same likely to be influenced by the hydraulic irreversibility. Figure 15(b) plots the same complete drying wetting path in the $\left(s-p^{\prime}\right)$ plane. Due to the inclusion of product of suction by degree of saturation inside the effective stress formulation, the hydraulic hysteresis is naturally featured. In consequence, even with the same yield limit, plastic states could be reached sooner or later according to the process applied, which might result in relevant simplifications in the constitutive formulation.

Indeed, this natural repercussion of the soil water retention curve shape can be obtained only if the mechanical stress variable accounts for both suction and degree of saturation, which is verified only in category 2 of effective stresses. Other stress frameworks, for instance, with effective stress 

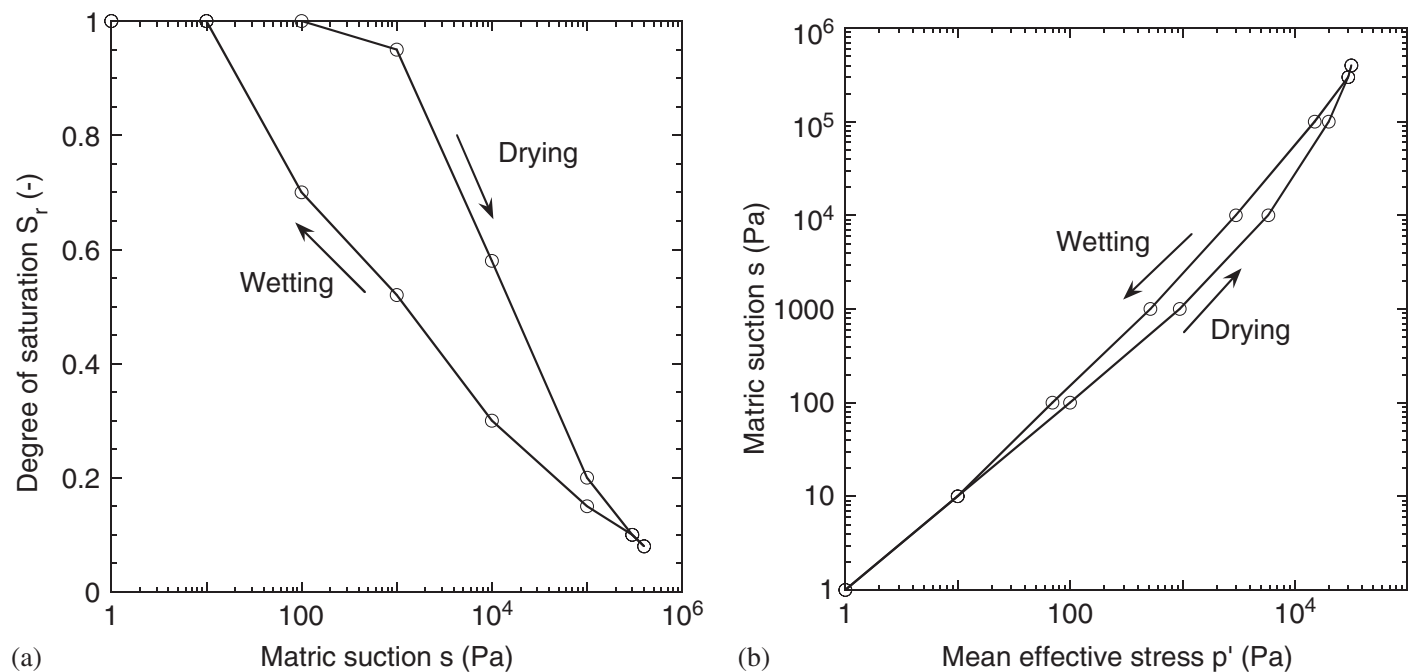

Figure 15. (a) Soil water retention curve of Jossigny loam (after [53]) and (b) Corresponding stress paths in $\left(s-p^{\prime}\right)$ plane.

based on suction ratio, have to be combined with advanced coupled elasto-plastic contexts in order to approach hydraulic hysteresis effects within the mechanical part of the model.

\section{CONCLUSION}

The historical developments of the effective stresses have been reviewed with the objective of determining a proper stress framework for constitutive modelling of unsaturated soils. Starting from the saturated state, it has been shown that under the assumption of incompressibility of grains and fluid, Terzaghi's effective stress (Equation (4)) is the unique stress state variable.

The extension of the effective stress principle to unsaturated soils is possible; however, the terminology of effective stress is quite misleading, provided that a second stress variable is always necessary to fully describe the soil behaviour. Work input characterization, among other methods, enables the determination of possible combinations of stresses to use. It is not compulsory for the two stresses to be independent. No matter the stress framework adopted, it should be used within a complete elasto-plastic framework, particularly for modelling suction hardening effects.

Similarly to Gens [9] it has been proposed to classify the possible stresses into three types: the independent stress approach, the category 1 effective stresses and the category 2 effective stresses. Table I sums up the major capabilities and consequences for each stress framework, studying the following points:

- The ease of representation of the stress paths, linked to the complexity of stress variables.

- The ability to shift from saturated to unsaturated states.

- The inclusion of hydraulic couplings in the stresses and effects of hydraulic hysteresis.

- The ability to model directly increase in strength. 
Table I. Comparative study of stress frameworks.

\begin{tabular}{lcccc}
\hline Category & Representation & $\begin{array}{c}\text { Saturated-unsaturated } \\
\text { transition }\end{array}$ & $\begin{array}{c}\text { Hysteresis and } \\
\text { hydraulic effects }\end{array}$ & $\begin{array}{c}\text { Direct accounting } \\
\text { of increase in strength }\end{array}$ \\
\hline Independent stress variables & + & - & - & - \\
Category 1 effective stresses & - & + & - & + \\
Category 2 effective stresses & - & + & + & + \\
\hline
\end{tabular}

The Bishop's generalized effective stress has been adopted as the single mechanical stress. Porosity is voluntarily not included in the stresses and strains variables in order to obtain directly the soil water retention curve $S_{\mathrm{r}}=\tilde{S}_{\mathrm{r}}(s)$ for the hydraulic part of the behaviour. The major implications of the proposed stress framework have been investigated on the basis of experimental data. Notably, the critical state is assumed unique for any suction level, and a single mechanical yield surface is sufficient. Other aspects like curvature of the stress path in $\left(s-p^{\prime}\right)$ plane upon hydraulic loading as well as accounting for the hydraulic hysteresis are also of interest.

\section{ACKNOWLEDGEMENT}

This work was supported by the Swiss State Secretariat for Education and Research SER, Grant OFES C03.0021-COST C15.

\section{REFERENCES}

1. Terzaghi K. The shearing resistance of saturated soils and the angle between the planes of shear. International Conference on Soil Mechanics and Foundation Engineering. Harvard University Press: Cambridge, MA, 1936; 54-56.

2. Skempton AW. Effective stress in soils, concrete and rocks. Pore Pressure and Suction in Soils. Butterworths: London, 1960; 4-16.

3. Nur A, Byerlee J. Exact effective stress law for elastic deformation of rocks with fluids. Journal of Geophysical Research 1971; 76(26):6414-6419.

4. Khalili N, Witt R, Laloui L, Vulliet L, Koliji A. Effective stress in double porous media with two immiscible fluids. Geophysical Research Letters 2005; 32(15):15309.

5. Lu N, Likos WJ. Suction stress characteristic curve for unsaturated soil. Journal of Geotechnical and Geoenvironmental Engineering 2006; 132(2):131-142.

6. Biot MA. Theory of elasticity and consolidation for a porous anisotropic soil. Journal of Applied Physics 1955; 26(2):182-185.

7. Suklje L. Rheological Aspects of Soil Mechanics. Wiley: New York, 1969.

8. Jardine RJ, Gens A, Hight DW, Coop MR. Developments in understanding soil behaviour. Advances in Geotechnical Engineering. The Skempton Conference. Thomas Telford: London, 2004; 103-206.

9. Gens A. Constitutive modelling: application to compacted soils. Unsaturated Soils. Balkema: Paris, 1995; 1179-1200.

10. Bishop AW. The principle of effective stress. Tecnisk Ukeblad 1959; 39:859-863.

11. Bishop A, Donald I. The experimental study of partly saturated soil in the triaxial apparatus. 5th International Conference on Soil Mechanics and Foundation Engineering, Paris, 1961; 13-21.

12. Zerhouni MI. Rôle de la pression interstitielle négative dans le comportement des sols—apllication au calcul des routes. Ph.D. Thesis, Ecole Centrale Paris, Paris, 1991.

13. Jennings JE. A revised effective stress law for use in the prediction of the behaviour of unsaturated soils. Pore Pressure and Suction in Soils. Butterworths: London, 1960; 26-30.

14. Bishop AW, Alpan I, Blight GE, Donald IB. Factors controlling the strength of partly saturated cohesive soils. Conference Shear Strength Cohesive Soils. American Society of Civil Engineers: New York, 1960; 503-532. 
15. Jennings JEB, Burland JB. Limitations to the use of effective stresses in partly saturated soils. Géotechnique 1962; 12:125-144.

16. Schrefler BA. The finite element method in soil consolidation (with applications to surface subsidence). Ph.D. Thesis. University College of Swansea, 1984.

17. Jommi C. Remarks on the constitutive modelling of unsaturated soils. Experimental Evidence and Theoretical Approaches in Unsaturated Soils; Proceedings of an International Workshop, Trento, 2000; 139-153.

18. Aitchison GD. Relationships of moisture stress and effective stress functions in unsaturated soils. Pore Pressure and Suction in Soils. Butterworths: London, 1960.

19. Khalili N, Geiser F, Blight GE. Effective stress in unsaturated soils: review with new evidence. International Journal of Geomechanics 2004; 4(2):115-126.

20. Coleman JD. Stress/strain relations for partly saturated soils. Géotechnique 1962; 12:348-350 (Correspondence).

21. Khalili N, Khabbaz MH. A unique relationship for $x$ for the determination of the shear strength of unsaturated soils. Géotechnique 1998; 48(5):681-687.

22. Matyas EL, Radhakrishna HS. Volume change characteristics of partially saturated soils. Géotechnique 1968; 18:432-448.

23. Sivakumar V. A critical state framework for unsaturated soils. Ph.D. Thesis. University of Sheffield, Sheffield, 1993.

24. Leonards GA. Correspondence. Géotechnique 1962; 12:354-355.

25. Fredlund DG, Morgenstern NR. Stress state variables for unsaturated soils. Journal of the Geotechnical Engineering Division (ASCE) 1977; 103(GT5):447-466.

26. Tarantino A, Mongiovì L. Experimental investigations on the stress variables governing unsaturated soil behaviour at medium to high degrees of saturation. Experimental Evidence and Theoretical Approaches in Unsaturated Soils; Proceedings of an International Workshop, Trento, 2000; 3-18.

27. Alonso EE, Gens A, Josa A. A constitutive model for partially saturated soils. Geotechnique 1990; 40(3):405-430.

28. Geiser F, Laloui L, Vulliet L. Modelling the behaviour of unsaturated silt. Experimental Evidence and Theoretical Approaches in Unsaturated Soils; Proceedings of an International Workshop, Trento, 2000; 155-175.

29. Fredlund DG, Morgenstern NR, Widger RA. The shear strength of unsaturated soils. Canadian Geotechnical Journal 1978; 15:313-321.

30. Wheeler SJ, Sivakumar V. An elasto-plastic critical state framework for unsaturated soil. Géotechnique 1995; 45(1):35-53.

31. Geiser F, Laloui L, Vulliet L. Elasto-plasticity of unsaturated soils: laboratory test results on a remoulded silt. Soils and Foundations 2006; 46(4):545-556.

32. Houlsby GT. The work input to an unsaturated granular material. Géotechnique 1997; 47(1):193-196.

33. Dangla P, Coussy O. Approche énergétique du comportement des sols non saturés. In Mécanique des sols non saturés, Coussy O, Fleureau J-M (eds). Hermes Science Publications: Paris, 2002.

34. Coussy O. Poromechanics. Wiley: New York, 2004.

35. Hutter K, Laloui L, Vulliet L. Thermodynamically based mixture models of saturated and unsaturated soils. Mechanics of Cohesive-Frictional Materials 1999; 4:295-338.

36. Laloui L, Klubertanz G, Vulliet L. Solid-liquid-air coupling in multiphase porous media. International Journal for Numerical and Analytical Methods in Geomechanics 2003; 27:183-206.

37. Hassanizadeh SM, Gray WG. Mechanics and thermodynamics of multiphase flow in porous-media including interphase boundaries. Advances in Water Resources 1990; 13(4):169-186.

38. Muraleetharan KK, Wei CF. Dynamic behaviour of unsaturated porous media: governing equations using the theory of mixtures with interfaces (TMI). International Journal for Numerical and Analytical Methods in Geomechanics 1999; 23(13):1579-1608.

39. Kohgo Y, Nakano M, Miyazaki T. Theoretical aspects of constitutive modelling for unsaturated soils. Soils and Foundations 1993; 33(4):49-63.

40. Modaressi A, Abou-Bekr N. A unified approach to model the behavior of saturated and unsaturated soils. Computer Methods and Advances in Geomechanics. Balkema: Morgantown, 1994; 1507-1513.

41. Taibi S. Comportement mécanique et hydraulique des sols soumis à une pression interstitielle négative. Ph.D. Thesis, Ecole Centrale Paris, 1994.

42. Jommi C, Di Prisco C. Un semplice approcio teorico per la modellazione del comportamento meccanico di terreni granulari parcialmente saturi. Conference Il ruolo dei fluidi nei problemi di ingegneria geotecnica, Mondovi, 1994; 167-188.

43. Bolzon G, Schrefler BA, Zienkiewicz OC. Elastoplastic soil constitutive laws generalized to partially saturated states. Geotechnique 1996; 46(2):279-289. 
44. Sheng D, Sloan SW, Gens A. A constitutive model for unsaturated soils: thermomechanical and computational aspects. Computational Mechanics 2004; 33(6):453-465.

45. Tamagnini R. An extended cam-clay model for unsaturated soils with hydraulic hysteresis. Geotechnique 2004; 54(3):223-228.

46. Wheeler SJ, Sharma RS, Buisson MSR. Coupling of hydraulic hysteresis and stress-strain behaviour in unsaturated soils. Geotechnique 2003; 53(1):41-54.

47. Gallipoli D, Gens A, Sharma R, Vaunat J. An elasto-plastic model for unsaturated soil incorporating the effects of suction and degree of saturation on mechanical behaviour. Geotechnique 2003; 53(1):123-135.

48. Pereira J-M, Wong H, Dubujet P, Dangla P. Adaptation of existing behaviour models to unsaturated states: application to CJS model. International Journal for Numerical and Analytical Methods in Geomechanics 2005; 29(11):1127-1155.

49. Laloui L, Nuth M. An introduction to the constitutive modelling of unsaturated soils. Revue Européenne de Génie Civil 2005; 9(5-6):651-669.

50. Cui YJ, Delage P. Yielding, plastic behaviour of an unsaturated compacted silt. Geotechnique 1996 46(2):291-311.

51. Maâtouk A, Leroueil S, La Rochelle P. Yielding and critical state of collapsible unsaturated silty soil. Géotechnique 1995; 45(3):465-477.

52. Laloui L, Geiser F, Vulliet L. Constitutive modelling of unsaturated soils. Revue française de génie civil 2001; 5(6):797-807.

53. Fleureau JM, Kheirbeksaoud S, Soemitro R, Taibi S. Behavior of clayey soils on drying wetting paths. Canadian Geotechnical Journal 1993; 30(2):287-296.

54. Gray W, Schrefler BA. Thermodynamic approach to effective stress in partially saturated porous media. European Journal of Mechanics and Solids 2001; 20(4):521-538. 